The Astronomical Journal, 127:1513-1530, 2004 March

(C) 2004. The American Astronomical Society. All rights reserved. Printed in U.S.A.

\title{
THE INTEGRATED SPECTRUM OF M67 AND THE SPECTROSCOPIC AGE OF M32
}

\author{
Ricardo P. Schiavon \\ UCO/Lick Observatory, University of California, Santa Cruz, 1156 High Street, Santa Cruz, CA 95064; ripisc@ucolick.org \\ Nelson Caldwell \\ Smithsonian Astrophysical Observatory, 60 Garden Street, Cambridge, MA 02138; caldwell@cfa.harvard.edu \\ AND \\ JAMES A. Rose \\ Department of Physics and Astronomy, CB 3255, University of North Carolina, Chapel Hill, NC 27599; jim@physics.unc.edu \\ Received 2003 July 3; accepted 2003 December 3
}

\begin{abstract}
We construct an integrated spectrum of the intermediate-age, solar-metallicity Galactic cluster M67, from individual spectroscopic observations of bona fide cluster members. The spectrum so obtained is used as a template to test our stellar population synthesis models, in an age and metallicity regime where such models remain largely untested. As a result, we demonstrate that our models predict a spectroscopic age of $3.5 \pm 0.5$ Gyr for M67, which is the same age we obtain from fitting isochrones to the color-magnitude diagram of the cluster. Full consistency is reached when using either $\mathrm{H} \beta, \mathrm{H} \gamma$, or $\mathrm{H} \delta$ as the age indicator. We also check if the models, when applied to the cluster integrated spectrum, predict elemental abundances in agreement with the known detailed abundance pattern of the cluster. The models also pass the latter test, by predicting the abundances of iron, magnesium, carbon, and nitrogen in agreement with detailed abundance analyses of cluster stars to within 0.1 dex. Encouraged by the high degree of consistency of our models, we apply them to the study of the integrated spectrum of the central $3^{\prime \prime}$ of the compact elliptical galaxy M32. The resulting luminosityweighted age of the galaxy ranges between 2 and $3.5 \mathrm{Gyr}$, depending on the age indicator adopted. According to our models, the center of M32 seems to have a supersolar iron abundance, ranging between $[\mathrm{Fe} / \mathrm{H}] \sim+0.1$ and +0.3 , depending on the spectral index adopted. The light element magnesium seems to be underabundant in the center of M32 relative to iron by about $\sim 0.1-0.2$ dex, whereas the data are consistent with nearly solar carbon and nitrogen abundances relative to iron. We find that single-age, singlemetallicity stellar population models with solar-scaled abundance patterns cannot fit all the Balmer and metal lines in the integrated spectrum of M32. In particular, there is a systematic trend in the sense that bluer absorption lines indicate a younger age and a higher metallicity. This slight inconsistency can be due either to (unaccounted for) abundance ratio effects on blue iron and Balmer line indices or to a spread of the ages of the stellar populations in M32. Current stellar population models cannot break this degeneracy at the level of accuracy required to address this problem.
\end{abstract}

Key words: galaxies: clusters: general — galaxies: individual (M32) — galaxies: stellar content — open clusters and associations: individual (M67) - stars: evolution

\section{INTRODUCTION}

With the commissioning of both wide-field spectroscopic survey instruments (2dF, Colless et al. 2001; Sloan Digital Sky Survey [SDSS], York et al. 2000) and deep multiaperture spectroscopy on large-aperture telescopes (e.g., DEEP survey, Davis et al. 2003; VIRMOS-VLT Deep survey, Le Fèvre et al. 2001), it is becoming increasingly important to reliably extract information concerning the stellar populations in galaxies from their integrated spectra.

Stellar population synthesis (SPS) provides the only tool to estimate the ages and metal abundances of stellar populations in distant, unresolved galaxies. The application of SPS models to the study of unresolved systems, however, needs to be preceded by extensive and detailed testing against the observations of nearby, resolved systems, for which the key stellar population (SP) parameters, such as age, metal abundances (i.e., metallicities and abundance ratios), and initial mass function (IMF) are known independently. To gain confidence that modelling of the integrated spectra of galaxies is sufficiently reliable to extract useful information about their luminosity-weighted mean ages and chemical compositions, it is often proposed that integrated light observations of star clusters should be used as a testing ground for the evolutionary synthesis models that form the backbone of integrated light analysis. Recently, different groups have pursued this aim, by comparing SPS model predictions with the observations of globular clusters with a range of SP parameters (Leonardi \& Rose 2003; Maraston et al. 2003; Beasley, Hoyle, \& Sharples 2002; Puzia et al. 2002; Schiavon et al. 2002a, 2002b).

Given the extensive evidence in recent work that the integrated light of early-type galaxies is often dominated by intermediate-age stellar populations (e.g., Trager et al. 2000; Kuntschner 2000; Caldwell, Rose, \& Concannon 2003), it has become of fundamental importance that SPS models be tested in the regime of intermediate age $(\sim 4 \mathrm{Gyr})$ and solar metallicity. Moreover, this is the range of SP parameters expected in early-type galaxies at moderately high redshifts $(z \sim 1)$. Most of the previous studies, however, have 
concentrated on Galactic globular clusters, thus being limited to ages older than 10 Gyr. More recently, Leonardi \& Rose (2003) and Beasley et al. (2002) have extended SPS model calibration efforts to younger ages by comparing model predictions with observations of clusters from the Large Magellanic Cloud (LMC). In general, good consistency was found between SPS ages and metallicities and those from the literature. However, the quality of cluster color-magnitude diagrams (CMDs) for age determinations, as well as the variety of isochrones used in the literature, make the precision and homogeneity of CMD ages for LMC clusters quite low, compared with what can be achieved with Galactic clusters. Moreover, it is difficult to check the metallicity scale of SP models using LMC clusters because they lack reliable metallicity determinations based on classical abundance analysis of high-resolution spectra of stellar members. To this date, such conditions are met only for Galactic clusters, which makes them the ideal templates for the calibration of SP models.

In order to cure this deficiency, we constructed an integrated spectrum of M67, an old ( 4 Gyr) Galactic open cluster with solar metallicity. The latter spectrum is used as a template for the calibration of the SPS models in the regime of intermediate age and solar metallicity. Our integrated spectrum was constructed by observing individual bona fide cluster members and co-adding their spectra weighting according to their magnitudes and an assumed IMF.

We present a detailed comparison of our SPS models to the above integrated spectrum of M67. We find that our model spectroscopic ages based on all Balmer lines from $\mathrm{H} \beta$ through $\mathrm{H} \delta$ agree with the CMD-based cluster age to within $0.5 \mathrm{Gyr}$. We also verify whether the models correctly predict elemental abundances from integrated spectra, by comparing them with the well-known abundances of M67 stars, taken from the literature. Again, our models pass the test by predicting correctly the abundances of key elements, like magnesium, carbon, and nitrogen.

Our spectrum of M67 is also compared with an integrated spectrum of the central $3^{\prime \prime}$ of M32, obtained with the same instrumental setup. We find that the two spectra look remarkably alike, indicating that the luminosity-weighted age and metal abundances of M32 are likely to be very close to those of M67.

The above results encouraged us to compare our model predictions with the integrated spectrum of another benchmark of stellar population studies, the compact elliptical galaxy M32. The resulting spectroscopic age is somewhere between 2 and $3.5 \mathrm{Gyr}$, depending on the Balmer line adopted in the analysis. In addition, depending on the iron feature used, the mean iron abundance of M32 results slightly above solar, or strongly supersolar. Analysis of other features, like $\mathrm{Mg} b$ and $\mathrm{CN}$ bands, shows that magnesium is underabundant relative to iron in the center of M32, while carbon and nitrogen have about solar-abundance ratios relative to iron.

In $\S 2$, we describe the selection of our sample of M67 members and the observations. In $\S 3$, we describe the computation of the integrated spectrum of M67. In $\S 4$, a brief description of the models is presented, and they are compared with both the CMD and the integrated spectrum of M67. The analysis of the integrated spectrum of M32 is presented in $\S 5$, and in $\S 6$ we summarize our conclusions.

\section{OBSERVATIONAL DATA}

\subsection{Sample Selection}

\subsubsection{M67 Stars}

To obtain a representative integrated spectrum of M67 built up from observations of individual stars in the cluster, spectra and photometry covering the entire CMD from the tip of the giant branch through the lower main sequence are required. Included as well must be giant branch (GB) "clump" stars, i.e., the core helium burning phase, as well as blue straggler (BS) stars. As a starting point, we used the CMD of Montgomery, Marschall, \& Janes (1993, hereafter MMJ) to produce a candidate list of 103 stars covering the CMD from the tip of the GB down to $1.5 \mathrm{mag}$ below the main-sequence turnoff (MSTO; $V \sim 14$ ), as well as several GB clump stars and number of BS stars. The stars were selected for observation strictly on the basis of their positions in the CMD. The cutoff at the fainter MS magnitudes is imposed by the combination of required spectral resolution and signal-to-noise ratio $(\mathrm{S} / \mathrm{N})$. As described below, we filled in the lower MS with observations of solar-abundance field dwarfs.

Naturally, there will be interlopers potentially contaminating the true M67 cluster members. We use four methods to filter out such interlopers. The first is to search for propermotion nonmembers, based on the study carried out by Sanders (1977). We find 16 stars in our sample with a propermotion membership probability of $30 \%$ or less. A second criterion is radial velocity. In addition to our own velocities, which have a $1 \sigma$ accuracy of $\pm 11 \mathrm{~km} \mathrm{~s}^{-1}$, many of our stars are observed by either Mathieu et al. (1986), with a precision of better than $1 \mathrm{~km} \mathrm{~s}^{-1}$, and by Scott, Friel, \& Janes (1995), with a precision similar to ours. We find that 13 stars have radial velocities inconsistent with membership in M67; six of these stars overlap with the proper-motion nonmembers.

A third way to discover nonmembers is if the metal abundance of the star is inconsistent with the near-solar composition of M67. Specifically, the MSTO color of M67 is similar to that of the Galactic thick-disk population (Carney, Latham, \& Laird 1989; Gilmore, Wyse, \& Jones 1995). In addition, M67 is located approximately $0.5 \mathrm{kpc}$ above the Galactic plane, and thus at the distance where one can expect significant contamination from the thick-disk MSTO. To identify thick-disk MSTO stars, we measure the Lick Fe5270 and $\mathrm{H} \beta$ indices (Worthey et al. 1994) for all of the stars in our sample, then find a mean relation between Fe5270 and $\mathrm{H} \beta$. Stars that deviate by more than $-0.4 \AA$ in Fe5270, for their $\mathrm{H} \beta$, are deemed to be metal-weak, with the threshold at $-0.4 \AA$ determined from the fit to $\mathrm{H} \beta$ versus $[\mathrm{Fe} / \mathrm{H}]$ given in Worthey et al. (1994). Altogether we find six stars in the M67 turnoff region that are metal-poor. Five of these stars are proper-motion nonmembers, and three of the stars are further confirmed as radial velocity nonmembers. Given that the majority of stars in our list are indeed cluster members, it is suggestive that the proper-motion and radial velocity nonmembers that we find in the turnoff region are also systematically metal-poor. Thus, we conclude that there is significant contamination of the observed CMD of M67 with thick-disk turnoff stars.

A fourth way to detect nonmembers is by identifying stars with anomalous positions in the M67 CMD based on their inferred absolute $V$ magnitude, assuming membership in M67, and comparing them with distances inferred from their surface gravities. Here we make use of a pair of spectral indexes, Sr II 
$\lambda 4077 / \mathrm{Fe}$ I $\lambda 4045$ and $\mathrm{H} \delta / \mathrm{Fe}$ I $\lambda 4045$, that have been used by Rose $(1984,1985 a, 1985 b)$ to discriminate surface gravity in stars. Briefly, the method relies on the gravity sensitivity of the ionization balance between singly ionized strontium and neutral iron, with the temperature sensitivity moderated by the strength of $\mathrm{H} \delta$. Using this procedure, we find five stars with anomalous CMD positions, three of which are proper motion and/or radial velocity nonmembers as well. The five cases are as follows. The star MMJ 6496 is positioned at $V=11.26$ and $B-V=0.62$ in the CMD and thus is located at low surface gravity if it is indeed a member of M67, and at an unusual location, i.e., well above the MSTO, but too red for a BS star. However, the surface gravity determined from the Sr II $\lambda 4077 / \mathrm{Fe}$ I $\lambda 4045$ index indicates that it is a dwarf, and its Fe5270 index indicates solar composition. Thus, it appears to be a relatively nearby ( $\sim 200$ pc away) solar composition thin disk dwarf. The star MMJ 5336 has $V=12.96, B-V=1.11$, placing it in an anomalously red region of the bottom of the subgiant branch. Its spectral characteristics are all consistent with a cool thick-disk (i.e., metal-poor) giant. The star MMJ 6034 has similar characteristics to MMJ 5336. In contrast, the stars MMJ 5571 and MMJ 5688 have the problem of lying blueward in color of the MSTO. MMJ 5571 has $V=$ 12.65 and $B-V=0.52$, while MMJ 5688 has $V=12.89$ and $B-V=0.45$. For both of these stars the $\mathrm{Sr}$ II $\lambda 4077 / \mathrm{Fe}$ I $\lambda 4045$ index indicates low surface gravity.

We have summarized the case for the nonmember stars in Table 1, where stellar identifications (following the nomen- clature of MMJ), coordinates, magnitudes, and colors are listed together with the criteria used to decide the nonmembership of each star. In Table 2, we list the stars found to be members of M67, which were used to construct the integrated spectrum of the cluster. In Figure 1, we display the M67 bona fide member stars in the CMD.

\subsubsection{Lower MS Field Stars}

With the instrumental setup available (see $\S 2.2$ ), we cannot easily go fainter than $V=14$, so we need to fill in the lower MS by observing field stars of roughly solar abundance. Since these later type stars all have lifetimes in excess of the age of the Galaxy, they are consequently unevolved. In addition, they will be seen to contribute only modestly to the integrated spectral indices in the blue. Thus, we do not consider that using field stars as surrogates for M67 members in the K dwarf regime produces any serious concerns. The stars adopted are listed in Table 3, where the $V$ magnitudes listed are not the stars' apparent magnitudes, but rather the magnitudes they would have at the distance of M67. The latter were computed using the Hipparcos parallaxes of the field stars and the distance modulus of the cluster, discussed in $\S 4.1$.

\subsubsection{Field M Giants}

Due to the decreasing lifetime as a star ascends the GB, representation of the upper GB of M67 is sparse. To remedy this situation, we have observed a number of cool field $\mathrm{M}$ giants, which were selected among stars from the Jones (1999)

TABLE 1

Stars Found Not to Be Members of M67

\begin{tabular}{|c|c|c|c|c|c|}
\hline $\begin{array}{c}\text { ID } \\
\text { (MMJ No.) }\end{array}$ & $\begin{array}{c}\text { R.A. } \\
\text { (J2000.0) }\end{array}$ & $\begin{array}{c}\text { Decl. } \\
(\mathrm{J} 2000.0)\end{array}$ & V & $B-V$ & Criteria $^{a}$ \\
\hline $6474 \ldots \ldots \ldots \ldots \ldots \ldots \ldots \ldots$ & 085021.1 & 122111.5 & 10.520 & 1.230 & 1 \\
\hline $6511 \ldots \ldots \ldots \ldots \ldots \ldots \ldots$ & 085156.0 & 115126.4 & 10.600 & 0.340 & 2 \\
\hline 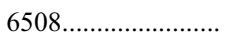 & 085210.9 & 113149.3 & 10.930 & 1.150 & 2 \\
\hline 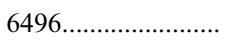 & 085146.0 & 113618.6 & 11.260 & 0.620 & $1,2,3$ \\
\hline 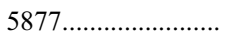 & 085130.4 & 114857.6 & 12.110 & 1.006 & 1 \\
\hline 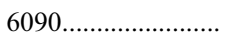 & 085142.6 & 114636.4 & 12.368 & 0.581 & $1,2,4$ \\
\hline 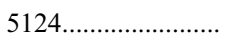 & 085041.5 & 114430.0 & 12.374 & 0.746 & 2,4 \\
\hline $6293 \ldots \ldots \ldots \ldots \ldots \ldots$ & 085158.5 & 114652.7 & 12.429 & 0.618 & 2,4 \\
\hline 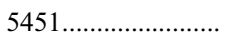 & 085107.2 & 115301.6 & 12.595 & 0.637 & 4 \\
\hline 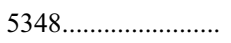 & 085100.8 & 113937.5 & 12.620 & 0.721 & 2 \\
\hline 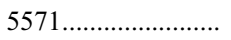 & 085115.4 & 114731.3 & 12.651 & 0.517 & 3 \\
\hline $5624 \ldots \ldots \ldots \ldots \ldots \ldots \ldots$ & 085117.9 & 114554.1 & 12.730 & 0.554 & 1 \\
\hline $5239 \ldots \ldots \ldots \ldots \ldots \ldots \ldots$ & 085051.7 & 114810.1 & 12.846 & 0.501 & 2 \\
\hline $5864 \ldots \ldots \ldots \ldots \ldots \ldots$ & 085129.4 & 115413.6 & 12.868 & 0.603 & 1 \\
\hline $5688 \ldots \ldots \ldots \ldots \ldots \ldots \ldots$ & 085120.5 & 114616.2 & 12.891 & 0.448 & 3 \\
\hline 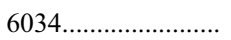 & 085137.9 & 115849.0 & 12.911 & 1.000 & 2,3 \\
\hline $5336 \ldots \ldots \ldots \ldots \ldots \ldots$ & 085059.7 & 113922.2 & 12.964 & 1.107 & $1,2,3$ \\
\hline $5222 \ldots \ldots \ldots \ldots \ldots \ldots \ldots$ & 085050.7 & 113503.0 & 12.988 & 0.540 & $1,2,4$ \\
\hline $6172 \ldots \ldots \ldots \ldots \ldots \ldots \ldots$ & 085150.6 & 113633.8 & 13.419 & 0.445 & 1,2 \\
\hline 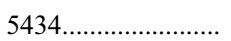 & 085107.4 & 113653.5 & 13.422 & 0.541 & 1 \\
\hline $6430 \ldots \ldots \ldots \ldots \ldots \ldots \ldots$ & 085213.4 & 114601.1 & 13.457 & 0.514 & 1 \\
\hline 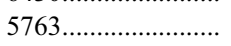 & 085124.1 & 114709.4 & 13.477 & 0.614 & 2 \\
\hline 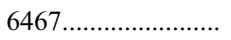 & 085216.6 & 114229.8 & 13.517 & 0.536 & 2 \\
\hline 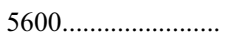 & 085117.6 & 113936.0 & 13.562 & 0.567 & 2 \\
\hline $5257 \ldots \ldots \ldots \ldots \ldots \ldots \ldots$ & 085053.4 & 114043.5 & 13.726 & 0.609 & $1,2,4$ \\
\hline 6009 & 085136.3 & 115650.5 & 14.001 & 0.617 & 1 \\
\hline
\end{tabular}

NoтE.-Units of right ascension are hours, minutes, and seconds, and units of declination are degrees, arcminutes, and arcseconds.

${ }^{a}$ Reasons for determination of nonmembership: (1) radial velocity; (2) proper motion; (3) position in CMD; (4) metallicity. 
TABLE 2

Stars Found to Be Members of M67 and Were Used in the Construction of the Cluster Integrated Spectrum

\begin{tabular}{|c|c|c|c|c|}
\hline $\begin{array}{c}\text { ID } \\
\text { (MMJ No.) }\end{array}$ & $\begin{array}{c}\text { R.A. } \\
\text { (B1950.0) }\end{array}$ & $\begin{array}{c}\text { Decl. } \\
\text { (B1950.0) }\end{array}$ & V & $B-V$ \\
\hline $6395 \ldots$ & 084925.57 & 115805.5 & 14.036 & 0.615 \\
\hline 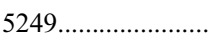 & 084809.05 & 115117.9 & 13.703 & 0.561 \\
\hline 5583 & 084832.24 & 115549.8 & 13.491 & 0.630 \\
\hline $6134 \ldots$ & 084902.38 & 115745.2 & 13.381 & 0.572 \\
\hline $5342 \ldots$ & 084814.97 & 120752.5 & 13.265 & 0.575 \\
\hline $5741 \ldots$ & 084838.86 & 120030.4 & 13.260 & 0.464 \\
\hline $5795 \ldots$ & 084841.37 & 120356.3 & 13.259 & 0.619 \\
\hline $5716 \ldots$ & 084837.99 & 115758.2 & 13.183 & 0.581 \\
\hline 5679......................... & 084836.28 & 115709.6 & 13.145 & 0.566 \\
\hline 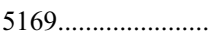 & $0848 \quad 02.00$ & 115421.7 & 13.117 & 0.542 \\
\hline 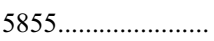 & 084845.32 & 115645.3 & 12.934 & 0.917 \\
\hline 6114 & 084900.70 & 115804.5 & 12.934 & 0.919 \\
\hline $5228 \ldots$ & 084805.79 & 120028.3 & 12.931 & 0.851 \\
\hline $6259 \ldots \ldots \ldots \ldots \ldots$ & $\begin{array}{lll}08 & 49 & 12.03\end{array}$ & 120133.9 & 12.920 & 0.983 \\
\hline $5969 \ldots \ldots \ldots \ldots$ & 084850.19 & 120101.7 & 12.909 & 0.534 \\
\hline $6169 \ldots$ & 084904.65 & 120810.1 & 12.906 & 0.970 \\
\hline $5688 \ldots$ & 084836.52 & 115733.6 & 12.891 & 0.448 \\
\hline $6408 \ldots$ & 084927.32 & 115657.3 & 12.889 & 0.817 \\
\hline $5318 \ldots$ & $0848 \quad 13.96$ & 120338.2 & 12.862 & 0.941 \\
\hline $5284 \ldots .$. & $0848 \quad 10.12$ & 120744.4 & 12.844 & 0.522 \\
\hline $5756 \ldots \ldots \ldots \ldots \ldots \ldots \ldots$ & 084839.74 & 115833.4 & 12.835 & 0.783 \\
\hline 5586......................... & 084831.59 & 120415.7 & 12.831 & 0.567 \\
\hline $5996 \ldots$ & 084851.19 & 120914.6 & 12.826 & 0.775 \\
\hline $5118 \ldots \ldots \ldots \ldots \ldots \ldots$ & 084756.63 & 115900.8 & 12.818 & 0.521 \\
\hline $5248 \ldots \ldots \ldots \ldots$ & $0848 \quad 07.54$ & 120811.0 & 12.803 & 0.547 \\
\hline 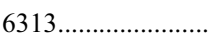 & 084915.49 & 120416.9 & 12.790 & 0.584 \\
\hline $5833 \ldots \ldots \ldots \ldots \ldots$ & 084844.05 & 120045.0 & 12.784 & 0.487 \\
\hline $5350 \ldots$ & $0848 \quad 15.96$ & 120548.0 & 12.782 & 0.813 \\
\hline $5927 \ldots$ & 084848.33 & 115919.0 & 12.777 & 0.822 \\
\hline $6107 \ldots$ & 084859.97 & 115742.9 & 12.750 & 0.758 \\
\hline $5362 \ldots$ & $\begin{array}{lll}08 & 48 & 16.91\end{array}$ & 120127.1 & 12.725 & 0.739 \\
\hline $5993 \ldots$. & 084851.97 & 115751.6 & 12.722 & 0.683 \\
\hline $5059 \ldots \ldots$ & 084752.01 & 115430.2 & 12.712 & 0.913 \\
\hline 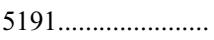 & $0848 \quad 03.55$ & 115605.6 & 12.700 & 0.482 \\
\hline $6228 \ldots \ldots \ldots \ldots \ldots$ & 084908.23 & 121541.8 & 12.688 & 0.622 \\
\hline 5853 ....................... & 084844.67 & $1203 \quad 17.6$ & 12.677 & 0.684 \\
\hline $5571 \ldots \ldots$. & 084831.36 & 115848.4 & 12.651 & 0.517 \\
\hline $5929 \ldots \ldots \ldots \ldots \ldots$ & 084848.38 & 115910.2 & 12.648 & 0.617 \\
\hline $5041 \ldots \ldots \ldots \ldots \ldots$ & 084750.31 & 115111.4 & 12.629 & 0.594 \\
\hline 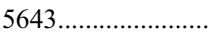 & 084834.61 & 115819.8 & 12.596 & 0.783 \\
\hline $5451 \ldots \ldots \ldots \ldots \ldots$ & 084823.04 & 120418.3 & 12.595 & 0.637 \\
\hline $5790 \ldots$ & 084841.30 & 115851.7 & 12.533 & 0.589 \\
\hline $6089 \ldots$ & 084858.36 & $1201 \quad 10.3$ & 12.514 & 0.598 \\
\hline 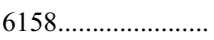 & 084904.22 & 120230.6 & 12.505 & 0.663 \\
\hline $5544 \ldots \ldots \ldots \ldots \ldots \ldots$ & 084829.47 & 120154.9 & 12.286 & 0.676 \\
\hline 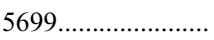 & 084837.17 & 115709.9 & 12.260 & 0.569 \\
\hline 5997 ....................... & 084851.62 & 120452.9 & 12.230 & 0.993 \\
\hline $5667 \ldots \ldots \ldots \ldots \ldots$ & 084835.84 & 115817.5 & 12.126 & 0.458 \\
\hline $6477 \ldots \ldots$ & $0848 \quad 11.49$ & 120330.3 & 12.04 & 0.60 \\
\hline $6502 \ldots \ldots \ldots \ldots \ldots$ & 084858.23 & 120126.0 & 11.63 & 1.05 \\
\hline $6484 \ldots .$. & 084823.70 & 115925.7 & 11.55 & 0.41 \\
\hline 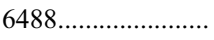 & 084839.66 & 120106.6 & 11.52 & 0.87 \\
\hline $6505 \ldots \ldots \ldots \ldots \ldots$ & 084858.23 & 120241.3 & 11.33 & 1.07 \\
\hline $6491 \ldots$ & 084837.60 & 120355.1 & 11.315 & 0.61 \\
\hline $6489 \ldots$ & 084832.90 & 120203.4 & 11.20 & 1.08 \\
\hline 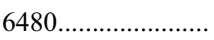 & 084830.30 & 115617.3 & 11.078 & 0.43 \\
\hline 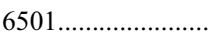 & 084848.51 & 120009.9 & 11.063 & 0.19 \\
\hline 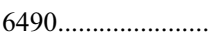 & 084842.88 & 120310.1 & 10.99 & 0.11 \\
\hline 6497..................... & 084859.52 & 115544.9 & 10.76 & 1.13 \\
\hline $6510 \ldots$ & 084926.73 & 115525.2 & 10.70 & 0.11 \\
\hline $6492 \ldots$ & $0848 \quad 28.53$ & 120359.1 & 10.59 & 1.12 \\
\hline $6506 \ldots \ldots \ldots \ldots \ldots$ & 084859.68 & 120800.8 & 10.58 & 1.10 \\
\hline 6512........................ & $\begin{array}{lll}08 & 49 & 15.35\end{array}$ & 120624.0 & 10.55 & 1.10 \\
\hline
\end{tabular}

TABLE 2-Continued

\begin{tabular}{|c|c|c|c|c|}
\hline $\begin{array}{c}\text { ID } \\
\text { (MMJ No.) }\end{array}$ & $\begin{array}{c}\text { R.A. } \\
\text { (B1950.0) }\end{array}$ & $\begin{array}{c}\text { Decl. } \\
\text { (B1950.0) }\end{array}$ & $V$ & $B-V$ \\
\hline 6503. & 084844.87 & 120150.7 & 10.55 & 1.12 \\
\hline 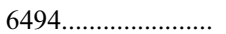 & 084842.01 & 120509.4 & 10.48 & 1.10 \\
\hline $6485 \ldots$ & 084838.71 & 115919.0 & 10.48 & 1.11 \\
\hline 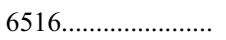 & 084934.57 & 115546.7 & 10.47 & 1.12 \\
\hline 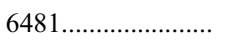 & 084827.72 & 115638.8 & 10.03 & -0.073 \\
\hline 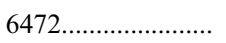 & 084734.04 & 120634.9 & 9.98 & 1.10 \\
\hline
\end{tabular}

spectral library, on the basis of the strength of TiO bands in their spectra. The stars adopted are listed in Table 3.

\subsection{Observations}

Stars in M67 were observed from 2002 January to 2002 April, using the FAST spectrograph (Fabricant et al. 1998) on the $1.5 \mathrm{~m}$ telescope of the Whipple Observatory. A 600 grooves $\mathrm{mm}^{-1}$ grating was used with a $2^{\prime \prime}$ slit, which gave a spectral coverage from 3500 to $5500 \AA$, at a resolution of $2.7 \AA$ and a dispersion of $0.75 \AA$ pixel $^{-1}$. All observations were taken with the slit aligned with the parallactic angle. Exposure times ranged from a few seconds for the bright giants to 30 minutes for MS stars. Spectra were extracted from the CCD images in the standard way using programs in IRAF and fluxcalibrated using standard-star spectra collected during the observing run. Nonphotometric conditions and the relatively small slit meant that the zero point of the flux calibration is not accurate, although the relative fluxes between wavelengths

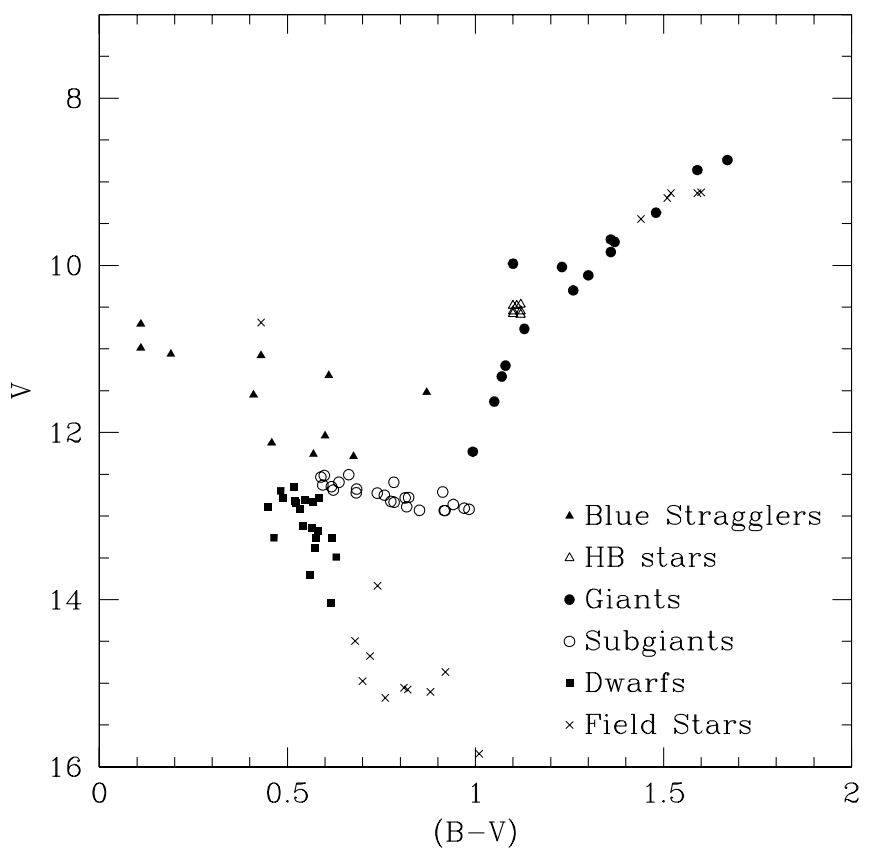

Fig. 1.-CMD showing the M67 members and field stars observed in this work. The photometry of M67 stars was taken from MMJ. Our observations of M67 members cover all the evolutionary stages of relevance for integrated light studies in the optical. Different evolutionary stages are denoted by different symbols, indicated in the bottom right corner. For completeness, we also observed field stars in areas where the CMD of M67 is scarcely populated (M giants) or where the stars are too faint to be observed efficiently (K dwarfs). Photometry of field stars was taken from the SIMBAD database, together with their Hipparcos parallaxes. The latter data were used to estimate the stars' positions in the CMD as if they were at the distance of M67. 
TABLE 3

Field Stars Included in the Construction of the Integrated Spectrum of M67

\begin{tabular}{|c|c|c|c|}
\hline ID & $V$ & $B-V$ & Spectral Type \\
\hline 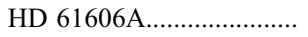 & 16.055 & 1.00 & $\mathrm{~K} 2 \mathrm{~V}$ \\
\hline HD 136834 . & 15.845 & 1.03 & $\mathrm{~K} 3 \mathrm{~V}$ \\
\hline 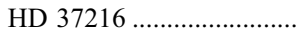 & 15.175 & 0.78 & G5 \\
\hline 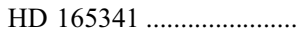 & 15.105 & 0.90 & $\mathrm{~K} 0 \mathrm{~V}$ \\
\hline 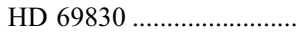 & 15.075 & 0.84 & $\mathrm{~K} 0 \mathrm{~V}$ \\
\hline 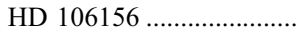 & 15.055 & 0.83 & G8 V \\
\hline 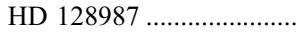 & 14.975 & 0.72 & G6 V \\
\hline 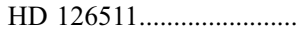 & 14.865 & 0.94 & G5 \\
\hline 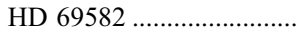 & 14.675 & 0.74 & G5 \\
\hline 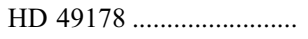 & 14.495 & 0.70 & G0 \\
\hline 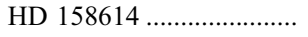 & 13.835 & 0.76 & G9 IV-V \\
\hline 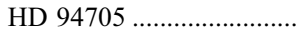 & 9.445 & 1.25 & M5.5 III \\
\hline 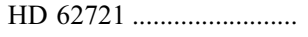 & 9.195 & 1.53 & K4 III \\
\hline 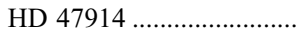 & 9.135 & 1.54 & K5 III \\
\hline 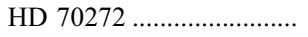 & 9.135 & 1.61 & K4.5 III \\
\hline 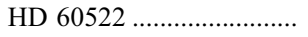 & 9.125 & 1.57 & M0 III \\
\hline HD 98991 .......................... & 10.685 & 0.45 & F3 IV \\
\hline
\end{tabular}

Note.-The $V$ magnitudes listed are the apparent magnitudes these stars would have if they were at the distance of M67.

should be good to about $5 \%$, based on the fits to all the spectrophotometric standard stars.

Bright field stars were observed with the same equipment and technique to supply a library for the fainter MS stars, as well as $\mathrm{M}$ giants.

We also obtained a spectrum of M32 using the same instrumental setup as above, but adopting a $3^{\prime \prime}$ slit width. The integrated spectrum was obtained through standard procedures, adopting an extraction window of $3^{\prime \prime}$ centered on the peak of the galaxy's light profile along the slit.

\subsubsection{Conversion to the Lick/IDS System}

In order to compare measurements of the equivalent widths (EWs) of absorption lines in our observed spectra with model predictions, we need to convert our measurements into the Lick/IDS system. The standard recipe for this calibration has been described in detail by Worthey \& Ottaviani (1997). It consists of collecting spectra of Lick/IDS standards (Worthey et al. 1994), smoothing those spectra to the Lick/IDS resolution (given by Worthey \& Ottaviani 1997), and comparing the EWs measured in the smoothed spectra with the standard values. In Figure 2, we plot the difference between the EWs of Lick indices measured in our standard-star spectra and those measured in the spectra of the same stars in the Jones (1999) spectral library, converted to the Lick/IDS system (for details, see Schiavon 2004).

Our measurements are generally in good agreement with the standard values for most of the indices. For some indices, we find a systematic trend of the residuals as a function of index strength. The most obvious case is that of $\mathrm{Ca} 4227$, but slight trends can also be seen for $\mathrm{H} \delta_{F}, \mathrm{G} 4300, \mathrm{Fe} 4383$, and $\mathrm{Mg}_{2}$. The errors in our index values were estimated from measurements made on 20 repeat spectra taken for seven Lick standards. In all cases, the error bars are about the same size as the symbols in Figure 2, indicating that most of the scatter in those plots comes from converting between EW systems defined by the different instrumental setups. We will henceforth base our error bars on the rms scatter between our data for Lick standards and the standard values (Fig. 2).
A key aspect of such calibrations is that the calibrating standards must encompass the range of EWs characteristic of the target systems, otherwise systematic effects such as that seen in Figure 2 cannot be adequately accounted for, possibly resulting in large errors. For this reason, we indicate by arrows in Figure 2 the index values measured in the spectra of the two targets of our analysis, M67 and M32.

For the indices not exhibiting any slope as a function of index strength, only minor zero-point corrections were sufficient to bring our measurements to the Lick/IDS system. That was the case for $\mathrm{CN} 1, \mathrm{CN} 2, \mathrm{H} \gamma_{F}, \mathrm{H} \beta, \mathrm{Mg} b, \mathrm{Fe} 5270, \mathrm{Fe} 5335$, and $\mathrm{Mg}_{2}$. For the remaining indices, the zero-point corrections were computed as the average value in a $\sim 2 \AA$ wide window around the values measured for M67 and M32 (0.1 mag in the case of $\mathrm{CN} 1, \mathrm{CN} 2$, and $\left.\mathrm{Mg}_{2}\right)$. On average, 15 stars were used in computing the zero-point corrections. We note that the index values for M67 and M32 are in regions of the index space that are very well populated by standard stars, so that we consider our calibration onto the Lick/IDS system to be robust.

It is important to emphasize how accurately such calibration needs to be done. For instance, a $\pm 0.1 \AA$ shift in $\mathrm{H} \delta_{F}$ implies a change in the spectroscopic age of M67 by about $\mp 1$ Gyr. $\mathrm{H} \beta$ and $\mathrm{H} \gamma$ are slightly less affected, but the case of the $\mathrm{G}$ band (G4300) is especially delicate. There is a sharp decline of the residuals for EWs larger than $\sim 5 \AA$, and as a result, if we had adopted a zero-point correction considering all the calibrating standards, the G4300-based spectroscopic age of M67 would be 2 Gyr older (see discussion in $\S 4.2 .2$ ).

In the Appendix, we provide a table with Lick/IDS index measurements taken in the spectra of all the field and M67 stars observed in this work.

\section{INTEGRATED SPECTRUM OF M67}

In this section, we describe the construction of the integrated spectrum of M67 from the individual spectra of the stellar members and field stars displayed in Figure 1. The reader who is not interested in technicalities, and would rather focus on the analysis of the integrated spectrum, is advised to skip to $\S 3.2$.

The integrated spectrum of the cluster is given by the following expression

$$
F(\lambda)=\sum_{i=1}^{N} f_{i}(\lambda) n_{i} \times 10^{-0.4\left(B_{i}-B_{i}^{\prime}\right)}
$$

where $f_{i}(\lambda)$ is the flux-calibrated spectrum of the $i$ th star and $B_{i}$ is its $B$ magnitude taken from the MMJ photometry. The magnitudes were corrected for interstellar reddening assuming $E(B-V)=0.05 . B_{i}^{\prime}$ is a magnitude measured in the observed spectrum by integrating it with a $B$-filter transmittance curve taken from Lejeune, Cuisinier, \& Buser (1997). The latter step was taken in order to normalize all the spectra in a similar fashion before weighting them according to the stars' dereddened magnitudes.

The quantity $n_{i}$ is proportional to the number of stars at each evolutionary stage. For all the stars from the MS to the tip of the first-ascent GB, $n_{i}$ was computed assuming a power-law IMF, normalized such that the total mass of the system is equal to $1 M_{\odot}$. For horizontal-branch stars and BSs, we followed a different procedure as explained a few paragraphs below.

In order to compute $n_{i}$, we need to estimate the stellar masses. This was achieved by interpolating stellar colors or magnitudes in a theoretical isochrone for solar metallicity and an age of 

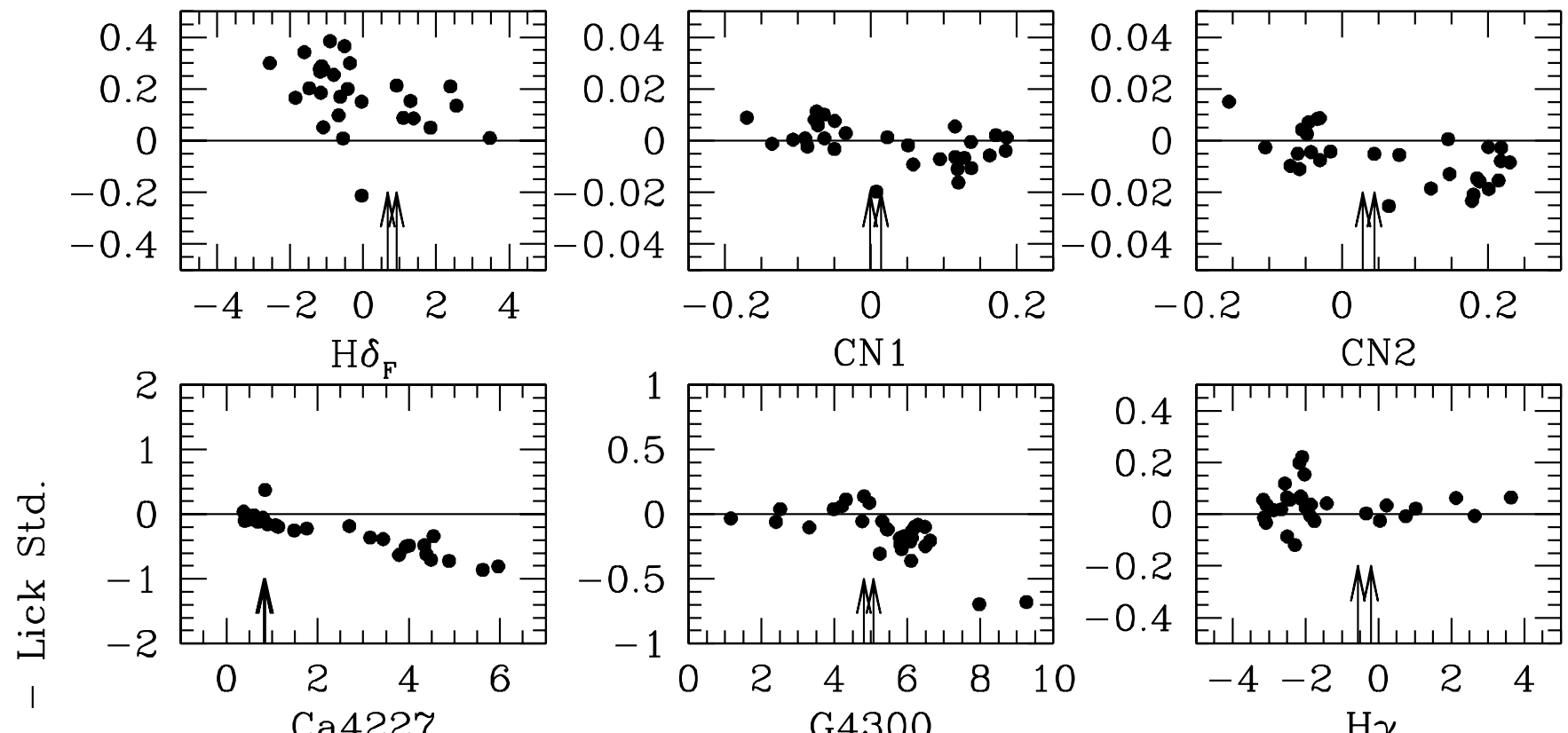

CN1
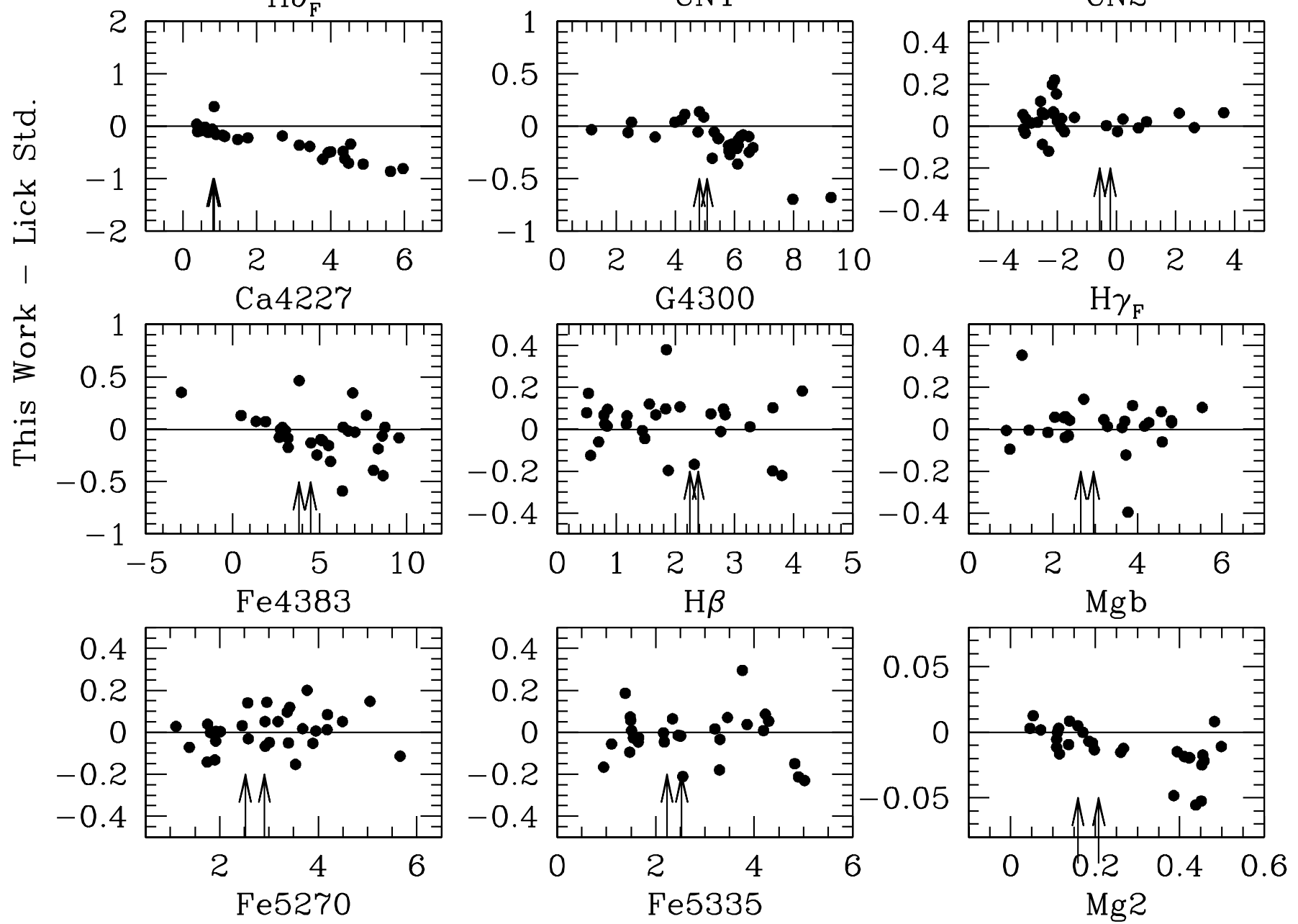

FIG. 2.-Comparison of the line indices measured in our spectra for Lick/IDS standards with the standard values. For all indices (except Ca4227) only minor zero-point corrections are needed to convert our data to the Lick/IDS system. The arrows indicate the values measured in the integrated spectra of M67 and M32, the main targets of this study, showing that they are in a locus in the index space that is very well populated by standard-star measurements. The error bars of our index measurements are about the same size as the symbols.

4 Gyr. The isochrone was truncated at the tip of the first-ascent GB. This procedure was followed for all stars except the M giants $\left(T_{\text {eff }}<4000 \mathrm{~K}\right)$. The stars are then sorted according to their masses so that the weight for each star was given by

$$
n_{i}=\int_{M_{i}-\left(\Delta M_{i}\right) / 2}^{M_{i}+\left(\Delta M_{i+1}\right) / 2} M^{-(1+x)} d M,
$$

where $M_{i}$ is the mass of the $i$ th star, $x$ is the IMF exponent, and $\Delta M_{i}=M_{i}-M_{i-1}$, so that the mass bin occupied by the $i$ th star is given by

$$
\Delta M=\left(M_{i+1}-M_{i-1}\right) / 2
$$

We adopt a Salpeter IMF, with $x=1.35$. It can be seen that the weight is higher for lower mass stars and higher for larger $\Delta M$. The latter means that an individual star in a more densely populated region of the CMD of Figure 1 has a lower weight in the final spectrum because the mass bin occupied by it is narrower. Along the GB, the mass varies by just a tiny fraction so that ultimately the weight for each star is mostly dependent on $\Delta M$. This is important for the reasons discussed in $\S 3.1$.

For BSs and horizontal-branch stars, $n_{i}$ was computed from the observed numbers relative to MS stars in the range $14.5<$ $m_{3890}<15.5$ as given by the CMDs of Fan et al. (1996) and Deng et al. (1999). This magnitude interval translates into 
$13.649<V<14.427$, according to the color transformations given by equations (3) and (4) of Fan et al.

Fan et al. (1996), analyzing photometric data complete down to many magnitudes below the turnoff, found that the presentday mass function of M67 has an exponent $x \sim 0.1$ for masses between 0.8 and $1.2 M_{\odot}$, and that it levels off for lower masses. They interpret this result as evidence that the cluster has undergone evaporation of low-mass stars. We tested our computations for the effect of the power-law exponent and found that both the overall spectral shape and the absorption-line EWs remain virtually constant even for wide variations of $x$. Therefore, throughout this paper, we adopt a single-exponent Salpeter IMF because our interest is in using M67 as a template single stellar population, free of the effects of dynamical evolution.

The assumed value for the reddening toward the cluster has a small impact in the computed integrated spectrum. This effect has been discussed by Schiavon et al. (2002a). It is due to the fact that a variation in $E(B-V)$ implies a change in the inferred absolute magnitudes of the stars, which in turn affects the computed stellar masses, and thus the relative weights given to each star in equation (1). The effect is small and is indicated in Figures 6 and 7 in the form of arrows.

\subsection{Contribution by M Giants to the Integrated Light}

For the M giants, the optical colors cannot be used for mass estimation for two reasons: $M$ giants are strongly variable, and optical colors saturate for temperatures below $4000 \mathrm{~K}$. The strong variability implies that a given $\mathrm{M}$ giant spectrum might not correspond to a measured color if they have not been collected at approximately the same time. On the other hand, saturation makes it difficult to use optical colors to get masses through interpolation in the isochrones. A good example is given by the pair of field stars HD 94705 (M5.5 III) and HD 62721 (K4 III). Their $B-V$ values are 1.46 and 1.53 , respectively, but the effective temperatures inferred from the strength of the TiO bands in their spectra are $\sim 3400$ and $3900 \mathrm{~K}$, respectively. If their colors were used to estimate their masses, and hence their weighting factors in equation (1), a large error would result in the case of HD 94705, because being cooler it represents a later, less populated evolutionary stage than HD 62721.

Our procedure was to estimate the $T_{\text {eff }}$ values of all stars redder than $B-V=1.3$ from the strength of their $\mathrm{TiO}$ bands, using an empirical calibration derived from measurements in the spectra of Lick standards whose $T_{\text {eff }}$ values were determined from angular diameter measurements or the infrared flux method (Schiavon 2004). The $T_{\text {eff }}$ values so derived are listed in Table 4 . They were used to estimate stellar masses

TABLE 4

Cool Giants Included in the Synthesis of the Integrated Spectrum of M67 and $T_{\text {eff }}$ Values Used to Estimate their Masses

\begin{tabular}{|c|c|c|c|}
\hline ID & $\begin{array}{l}T_{\text {eff }} \\
(\mathrm{K})\end{array}$ & ID & $\begin{array}{l}T_{\text {eff }} \\
(\mathrm{K})\end{array}$ \\
\hline 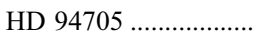 & 3350 & 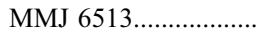 & 3950 \\
\hline 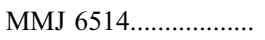 & 3740 & 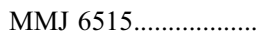 & 3950 \\
\hline 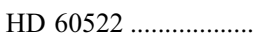 & 3900 & MMJ 6486...................... & 3950 \\
\hline 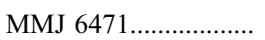 & 3920 & 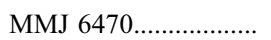 & 3960 \\
\hline 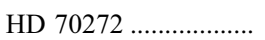 & 3930 & MMJ 6499...................... & 3960 \\
\hline HD 47914 & 3940 & MMJ $6482 \ldots \ldots \ldots \ldots \ldots$ & 3960 \\
\hline HD $62721 \ldots \ldots$ & 3940 & MMJ $6495 \ldots \ldots \ldots$ & 3960 \\
\hline
\end{tabular}

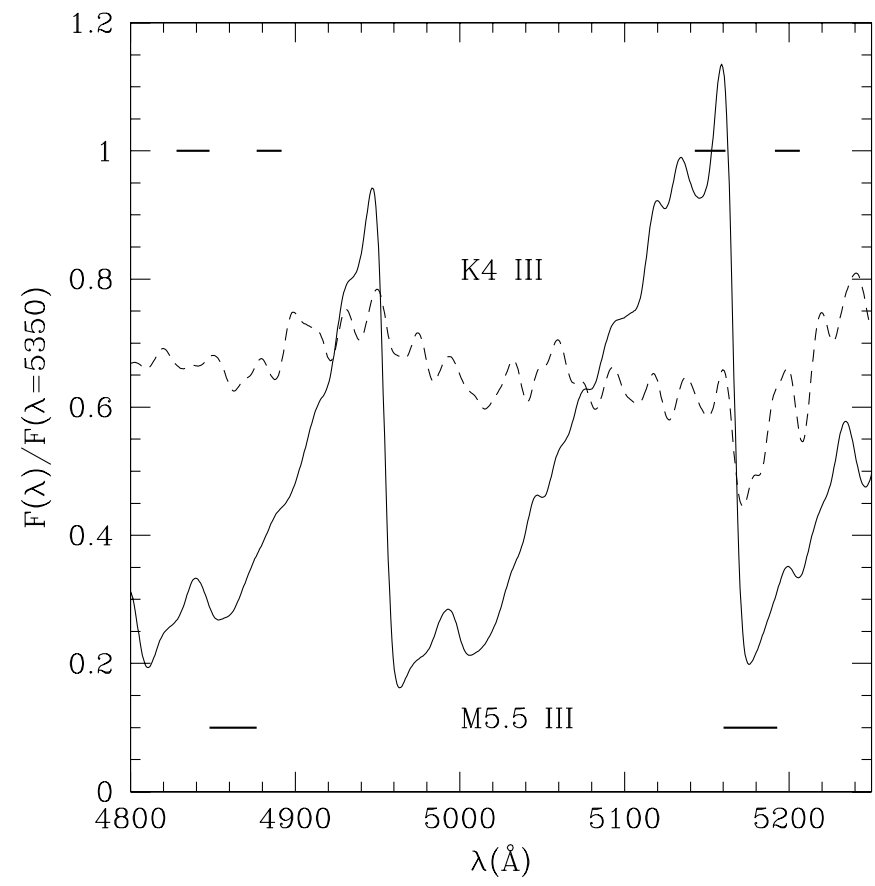

FIG. 3.-Comparison between the spectra of two giants: HD 94705 (M5.5 III, solid line) and HD 62721 (K4 III, dashed line), with essentially identical $B-V$ values. The pseudocontinua and passband of $\mathrm{H} \beta$ (left) and $\mathrm{Mg} b$ (right) are illustrated by the horizontal lines. The spectrum of the $\mathrm{M}$ giant is characterized by very strong TiO bands, whose band heads coincide with the passbands and continuum windows of the Lick indices. The case of $\mathrm{Mg} b$ is particularly sensitive, since $M$ giant stars contribute roughly 6 times more flux in the blue pseudocontinuum than in the index passband. For this reason, errors in estimating the contribution of very cool stars to the integrated light of M67 can cause sizable errors in the integrated value of $\mathrm{Mg} b$. The same comments apply, to a lesser extent, to $\mathrm{H} \beta$.

and colors for stars cooler than $\sim 4000 \mathrm{~K}$ through interpolation in the theoretical isochrone for $3.5 \mathrm{Gyr}$ and $[\mathrm{Fe} / \mathrm{H}]=0.0$. The weights of the $\mathrm{M}$ giants resulting from this procedure were substantially higher than when the masses were interpolated from their $B-V$ values, because there are only four $\mathrm{M}$ giants in our sample, and therefore each of these stars occupies a wide mass bin (eqs. [2] and [3]). As a result, when the $\mathrm{M}$ giants are accounted for in this way, the strength of the redder indices, especially $\mathrm{Mg} b$, becomes considerably stronger. In fact, the latter index becomes $\sim 0.3 \AA$ stronger than when the masses of $\mathrm{M}$ giants were (wrongly) estimated from their colors. $\mathrm{H} \beta$ is also affected, but to a lesser degree.

The dependence of $\mathrm{Mg} b$ and $\mathrm{H} \beta$ on the contribution by $\mathrm{M}$ giants is due to the very strong $\mathrm{TiO}$ bands present in their spectra, which can sizably affect the redder Lick indices. To illustrate this, we compare in Figure 3 the spectra of HD 62721 and HD 94705, overlaid by horizontal lines indicating the passband and pseudocontinuum definitions of $\mathrm{H} \beta$ and $\mathrm{Mg} b$. As can be seen, strong TiO band heads coincide with the index passbands. In particular, in the case of $\mathrm{Mg} b$, the $\mathrm{M}$ giant spectrum is roughly 6 times brighter in the blue pseudocontinuum window than in the index passband. Therefore, increasing the contribution by $\mathrm{M}$ giants to the integrated light causes a substantial increase in $\mathrm{Mg} b$. As a consequence, it is very important to compute the weights of the $M$ giants precisely, because even though their contribution to the total integrated light in the optical is not very large, their influence on key spectral indices like $\mathrm{Mg} b$ and $\mathrm{H} \beta$ is by no means negligible. It goes without saying that SP model predictions for 
such indices are likewise afflicted by such uncertainties. This is an issue that is seldom appreciated in the literature, in particular by stellar population modelers. The situation is, of course, even more serious for indices located toward the far red, where the contribution of $\mathrm{M}$ giants to the integrated light is dominant (e.g., Schiavon, Barbuy, \& Bruzual 2000; Schiavon \& Barbuy 1999).

Another important issue regards the luminosity function in the upper GB. In particular, our procedure to compute the integrated spectrum of M67 needs to account for stars in the asymptotic GB (AGB). Recall that when we computed the weights for the stars in equation (1), we truncated the isochrone used in the computation of stellar masses at the tip of the firstascent GB. In order to account for the AGB stars, we increase $n_{i}$ for stars brighter than the horizontal branch by $0.1 \mathrm{dex}$, according to the theoretical luminosity function of the Padua isochrones.

\subsection{Resulting Integrated Spectrum and the Effect of BSs}

The resulting integrated spectrum of M67 is displayed in Figure 4, where we compare the spectra obtained with and without the contribution by BSs. As can be seen in this figure, the contribution to the integrated light due to BSs is very large: when BSs are included in the computation, the resulting spectrum is considerably bluer and has stronger Balmer lines and weaker metal lines. This result agrees with the finding by Deng et al. (1999), who modelled the cluster's integrated light using intermediate-band photometry and synthetic stellar spectra from the Kurucz spectral library. Landsman et al. (1998) assessed the relative contribution of BS stars to the integrated light of M67 in the ultraviolet and found that BSs dominate the light of the cluster at $\sim 1500 \AA$. It is important to stress that this result does not depend on any assumption as to the number ratio of BS to MS stars, since the latter number is

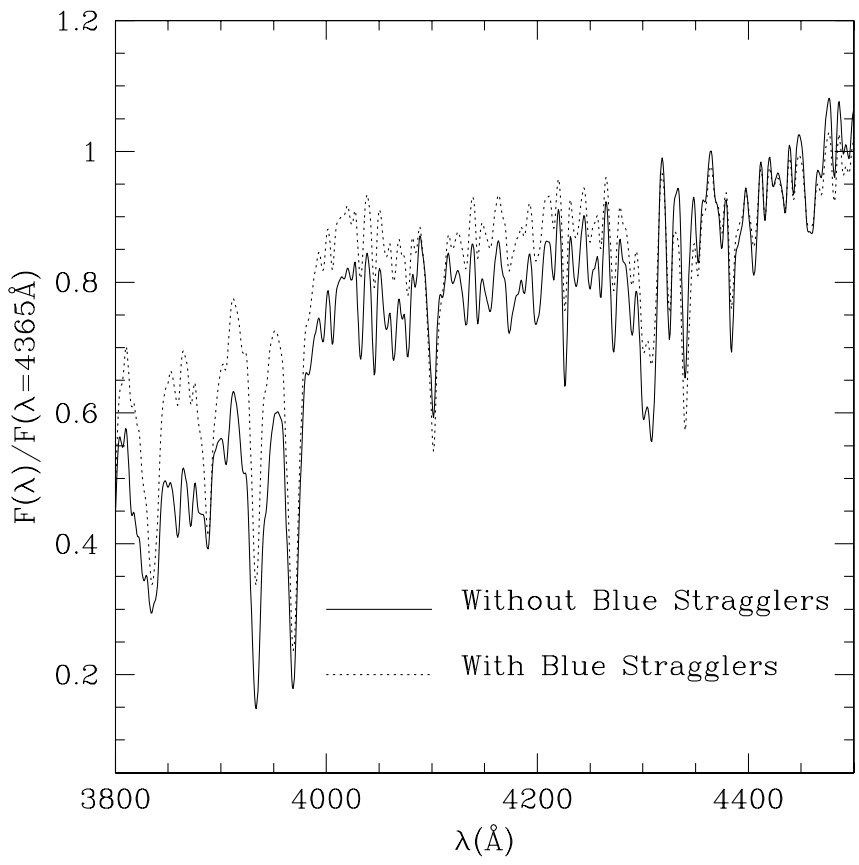

FIG. 4.- Integrated spectrum of M67, resulting from the computations described in $\S 3$. The spectrum represented by the solid line was obtained without considering the contribution due to the BSs. The spectrum obtained when the latter are included is shown as the dotted line. The contribution due to the BSs is very large. This is a robust result, since the number of BSs relative to MS stars is solidly constrained by photometry and membership studies. anchored on photometry and membership studies. Recall that our recipe to weigh BSs in number is based on the photometry by Fan et al., which is complete down to many magnitudes below the turnoff.

The BS frequency in M67 appears to be unusually high when compared with other clusters of similar age and richness (e.g., Ahumada \& Lapasset 1995; see also a discussion in Landsman et al. 1998). Hurley et al. (2001), using binary population synthesis and $N$-body simulations, propose that the high BS fraction in M67 is due to a combination of effects related to binary evolution, stellar encounters in the cluster high-density environment, and evaporation of low-mass stars.

Irrespective of the physical origin of the BS phenomenon, their mere existence is a potential source of confusion for those attempting to use Balmer lines to date stellar populations, as is clearly illustrated by the strength of Balmer lines when BSs are included in the integrated spectrum (Fig. 4). In fact, we show in $\S 4.2$ that the age one would infer from the integrated spectrum of M67 when BSs are included is substantially lower ( 1.2 Gyr). However, the case of M67 seems to be exceedingly unusual. As noted by Landsman et al. (1998), the central stellar density in the cluster is higher than that found in the centers of most nearby galaxies.

There is another reason to believe that such a high BS contribution does not seem to be a common phenomenon in the universe, and in particular in early-type galaxies, which are the ultimate targets of our modelling efforts. When BSs are included, the integrated spectrum of M67 has strong enough Balmer lines that it would be classified as a $k+a / a+k$ spectrum according to the definition of several different groups (Fisher et al. 1998; Balogh et al. 1999; Tran et al. 2003; Poggianti et al. 2003). Therefore, it is reasonable to assume that such a high fraction of BSs as found in M67, if common to the centers of early-type galaxies, would generate a high fraction of $k+a / a+k$ galaxies in the universe. However, recent estimates from the SDSS rate the fraction of $k+a / a+k$ galaxies in the local universe at slightly lower than $0.1 \%$ (Goto et al. 2003). We therefore conclude that a BS population as seen in M67 is, at most, extremely rare in field galaxies, and henceforth we concentrate on comparing our models with the integrated spectrum of M67 without the contribution of BSs. This is the topic of the next section.

The spectra shown in Figure 4 are helpful templates for the calibration of stellar population models in an age/metallicity regime where these models still remain largely untested. Therefore, we make them available electronically upon request to the authors.

\section{COMPARING STELLAR POPULATION MODELS WITH THE DATA FOR M67}

\subsection{Turnoff Age of $M 67$}

In this section, we estimate the age of M67 from the comparison of its CMD with theoretical isochrones. This is a very important step, since in the next section we will require our SP computations to be consistent with the ages inferred from the CMD, using the same set of isochrones in both procedures.

The iron abundance of M67 is about or slightly below solar (Brown 1987; Hobbs \& Thorburn 1991; Friel \& Boesgaard 1992; Tautvaisiene et al. 2000; Shetrone \& Sandquist 2000), and the abundance ratio between $\alpha$-elements and iron also seems to be nearly solar (Friel \& Boesgaard 1992; Shetrone \& Sandquist 2000; Tautvaisiene et al. 2000). Therefore, we 
adopt the isochrones by Girardi et al. (2000) for solar metallicity. Girardi et al.'s isochrones are computed for the solar mixture of heavy elements, and we will henceforth refer to them as the Padua isochrones.

In Figure 5, we display the color-magnitude data for M67 from MMJ, overplotted on the Padua isochrones for solar metallicity and ages of 2.8, 3.5, and 4.4 Gyr. The isochrones were transformed to the observational plane adopting the color versus $T_{\text {eff }}$ calibration described in Schiavon et al. (2002a) and the bolometric correction versus $T_{\text {eff }}$ calibration from Alonso, Arribas, \& Martínez-Roger (1995, 1999).

The reddening determinations for M67 range from $E(B-V)=$ 0.03 (Fan et al. 1996; Nissen, Twarog, \& Crawford 1987) to 0.05 (MMJ; Taylor 1980). We adopted a reddening of $E(B-V)=0.05$, within the range of previous determinations. The distance modulus adopted was $(m-M)_{0}=9.45$ (Chaboyer et al. 1999). The age error due to the uncertainty in the reddening toward the cluster is very small, mostly because at ages around 3-4 Gyr the color and luminosity of the turnoff are very agesensitive - as opposed to what happens for older ages (see discussion in Schiavon et al. 2002b). However, the uncertainties on the distance modulus and reddening do have some impact on the final integrated spectrum, as discussed in $\S 3$.

Deciding the exact position of the cluster's turnoff in Figure 5 is not obvious. There is a sparse group of stars between $V \sim 12.5$ and $\sim 12.0$ at $B-V \sim 0.6$ that seems to be well fitted by the "hook" above the turnoff of the $2.8 \mathrm{Gyr}$ isochrone. If these stars are not considered to be BSs, then the best-fitting cluster age would be $\sim 2.8$ Gyr. Conversely, if the "hook" is considered to be the clump of stars at $V \sim 12.7$ and $B-V \sim 0.6$, the $3.5 \mathrm{Gyr}$ isochrone would be a better fit. For consistency, we adopt the older age as the best fit, because the stars brighter than $V \sim 12.5$ were considered to be BSs when

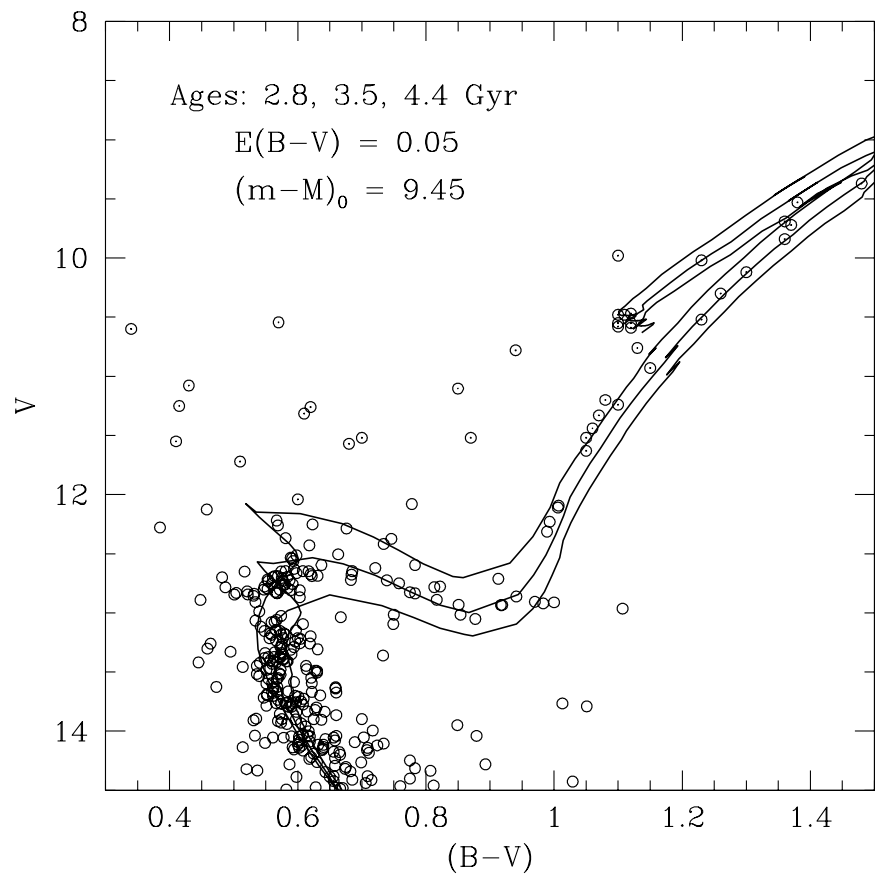

FIG. 5.-Comparison between the CMD of M67 (data from MMJ) and the isochrones from Girardi et al. (2000). The isochrones were transformed to the observational plane using the recipe described by Schiavon et al. (2002a) and the reddening and distance modulus indicated in the top left. From left to right: the ages of the theoretical isochrones are 2.8, 3.5, and 4.4 Gyr. The age that best fits the cluster data is $3.5 \mathrm{Gyr}$. we computed the integrated spectrum of the cluster (see Fig. 1). Therefore, for our present purposes, we adopt an age of 3.5 Gyr for M67. We note that Chaboyer, Green, \& Liebert (1999), analyzing the same photometric data, adopted a similar criterion to define the cluster turnoff, and find the same age for the cluster, even though they adopted a different set of isochrones and a different distance modulus. Most importantly, our age and distance modulus estimates are within the range of previous results in the literature: age $=3.5-5 \mathrm{Gyr}$, $(m-M)_{0}=9.35-9.60 \mathrm{mag}$ (see the review by Chaboyer et al. 1999 for details).

The assumption of convective core overshooting can also potentially affect age determinations in the intermediate-age regime ( $\lesssim 4 \mathrm{Gyr}$ ), where turnoff stars have masses $m \gtrsim 1.5 M_{\odot}$ (e.g., Maeder \& Meynet 1991). The isochrones by Girardi et al. (2000) include mild overshooting for stars with $m>1.5 M_{\odot}$ and a gradual decrease of the overshoot efficiency with decreasing mass down to $m=1 M_{\odot}$, where overshooting is turned off. The best-fitting isochrone in Figure 5 has a turnoff mass of $\sim 1.4 M_{\odot}$, which is within the regime of low overshooting efficiency in the Girardi et al. isochrones. Therefore, the age derived here should not be significantly dependent on the model prescription for overshooting.

Finally, we would like to stress that, for the purposes of this paper, it is not crucial to determine the absolute age of M67. Much more important for us is to focus on reaching consistency between spectroscopic and CMD-based ages. This is the topic of the next section.

\subsection{Comparison with Stellar Population Models}

\subsubsection{Models}

Before discussing the estimation of the age and metal abundances of M67 from its integrated spectrum, we recapitulate our modeling techniques.

The key ingredients of our models are a set of theoretical isochrones from the literature, a set of calibrations used to transform the latter into the observational plane, and a set of fitting functions, which are used to compute absorption line indices as a function of stellar parameters. The latter are integrated along the isochrones in order to produce integrated line indices of single stellar populations. The isochrones adopted here are the ones by Girardi et al. (2000), which were already presented in $\S 4.1$. The calibrations used to transform $T_{\text {eff }}$ and $M_{\text {bol }}$ into colors and magnitudes are a combination of those presented in Schiavon et al. (2002a) and the empirical calibrations by Alonso et al. (1995, 1999).

The fitting functions are the major new ingredient of our models. They are based on a redefinition of the Lick/IDS system, rooted in the Jones (1999) spectral library (a brief description can be found in Jones \& Worthey 1995), converted to the Lick/IDS resolution, and supplemented by further data from the original Lick/IDS library (Worthey et al. 1994). Most importantly, the fitting functions are based on a new set of homogeneous stellar parameters for the stars from the Jones spectral library. A detailed description of our stellar parameter determinations can be found in Schiavon et al. (2002a). A full description of the models, including a thorough comparison of our new fitting functions with those from the literature, will be presented elsewhere (Schiavon 2004).

\subsubsection{Spectroscopic Age of M67 According to the SP Models}

The absorption line indices measured in the integrated spectrum of M67 are listed in Table 5 and compared with the 
TABLE 5

Absorption Line Indices Measured

\begin{tabular}{|c|c|c|c|c|c|c|c|c|c|c|c|c|c|}
\hline Spectrum & $\mathrm{H} \delta_{F}$ & $\mathrm{H} \delta_{A}$ & CN1 & $\mathrm{CN} 2$ & G4300 & $\mathrm{H} \gamma_{F}$ & $\mathrm{H} \gamma_{A}$ & Fe4383 & $\mathrm{H} \beta$ & $\mathrm{Mg}_{2}$ & $\operatorname{Mg} b$ & Fe5270 & Fe5335 \\
\hline M67 (no BSs) ......... & 0.92 & -0.65 & 0.001 & 0.030 & 4.8 & -0.33 & -4.0 & 3.9 & 2.37 & 0.18 & 2.9 & 2.53 & 2.20 \\
\hline M67 (with BSs) ..... & 3.05 & 3.31 & -0.078 & -0.045 & 2.6 & 2.16 & 0.7 & 2.4 & 3.46 & 0.14 & 2.3 & 2.06 & 1.81 \\
\hline M32 ...................... & 0.75 & -1.12 & 0.015 & 0.046 & 4.9 & -0.56 & -4.5 & 4.6 & 2.25 & 0.21 & 3.0 & 2.95 & 2.60 \\
\hline Error ....................... & 0.09 & 0.14 & 0.005 & 0.008 & 0.1 & 0.05 & 0.2 & 0.2 & 0.09 & 0.01 & 0.1 & 0.06 & 0.08 \\
\hline
\end{tabular}

predictions of single stellar population models in Figures 6 and 7. The $1 \sigma$ error bars listed in Table 5 and adopted in all the figures involving integrated indices were estimated from the rms scatter between our measurements of Lick indices in Lick/IDS standard stars and the standard values (see Fig. 2). Assuming equal contribution to the rms scatter from our measurements and the standard values, we divide the rms by $\sqrt{2}$ to obtain the error estimates. These errors are certainly
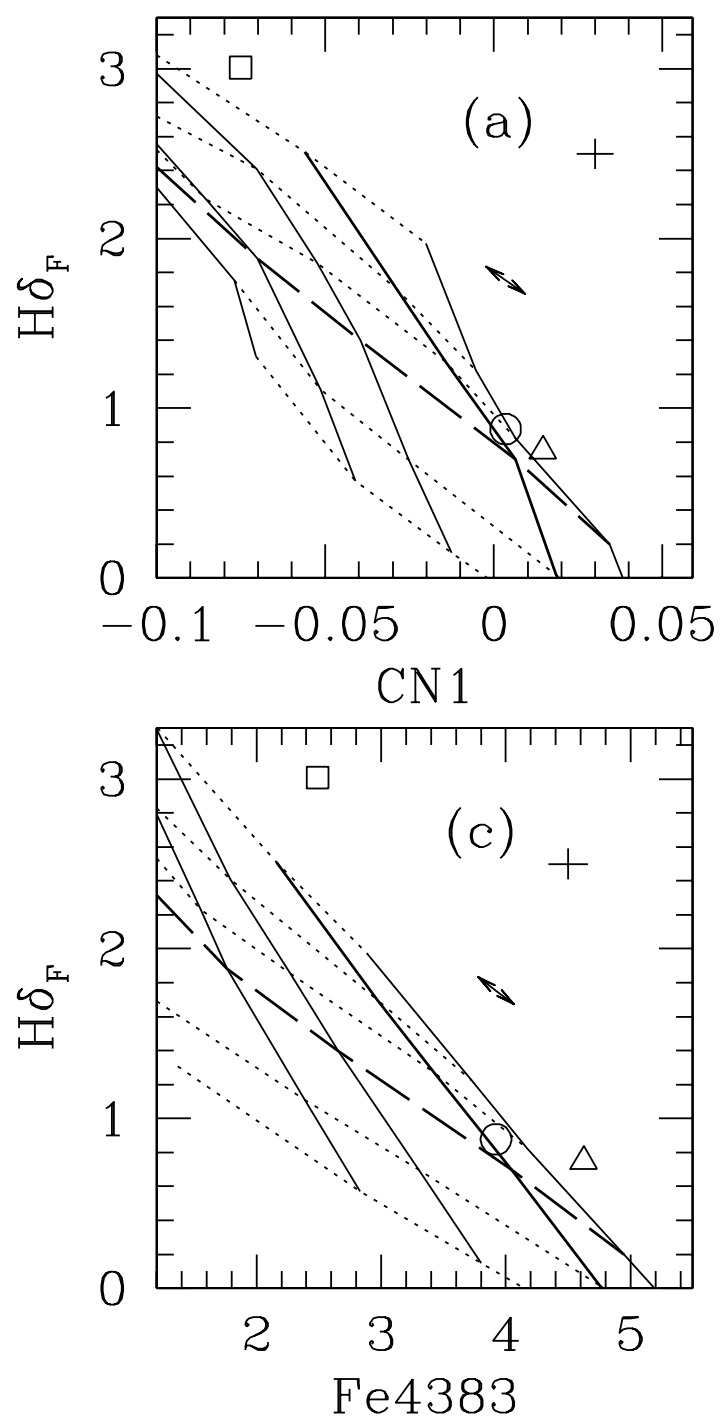

larger than expected from Poisson statistics and wavelength calibration errors, and so they are dominated by systematic effects of tying into the Lick/IDS system with spectra taken from different sources. Since the M32 and M67 composite spectra have very high $\mathrm{S} / \mathrm{N}$, comparable to those of the standard stars, we assume the same overall errors for M32 and M67.

We choose to compare the models with the observations using four age indicators $\left(\mathrm{H} \delta_{F}, \mathrm{G} 4300, \mathrm{H} \gamma_{F}\right.$, and $\left.\mathrm{H} \beta\right)$ and five

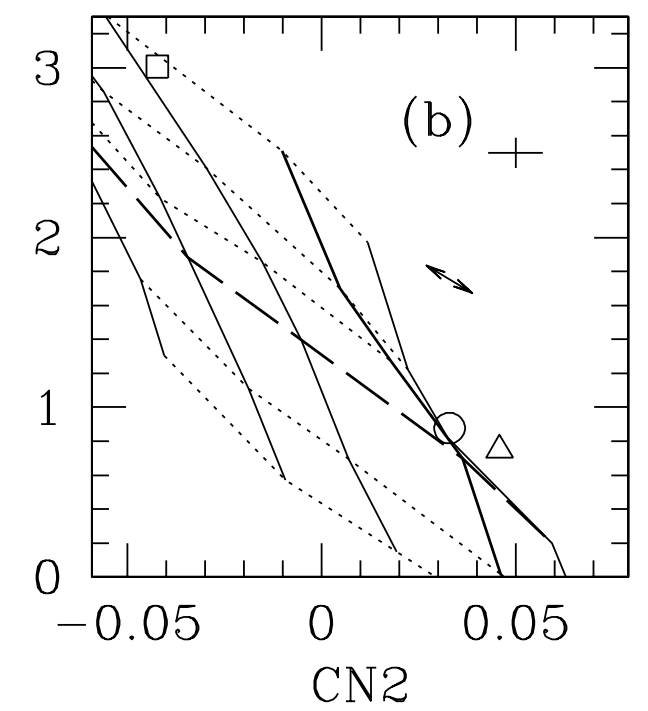

FIG. 6.-Comparison of model predictions absorption line indices for single stellar populations with those measured in the spectra of M32 (open triangle), M67 without BSs (open circle) and M67 including BSs (open square). Dotted lines connect model predictions for the same age. From top to bottom: 1.2 2.0, 2.5, 3.5, 7.9, and $14.1 \mathrm{Gyr}$. Solid lines connect model predictions for the same $[\mathrm{Fe} / \mathrm{H}]$. From right to left: $+0.2,0.0,-0.4,-0.7$, and -1.3 (in $[c]$, the line for $[\mathrm{Fe} / \mathrm{H}]=-1.3$ falls outside the plotting area). The lines for $[\mathrm{Fe} / \mathrm{H}]=0.0$ and age $=3.5 \mathrm{Gyr}$ are thicker, for clarity. The arrows indicate by how much the integrated indices of $\mathrm{M} 67$ vary for an error of \pm 0.015 in $E(B-V)$. A higher reddening makes Balmer lines look stronger and metal lines weaker. The spectroscopic age of M67, when BSs are not included in the integrates spectrum, is about $3.5 \mathrm{Gyr}$ in all diagrams, which is in agreement with the value inferred from the CMD. The data on M32 indicate that it has supersolar iron abundance and has a nearly solar abundance of carbon and/or nitrogen relative to iron. 

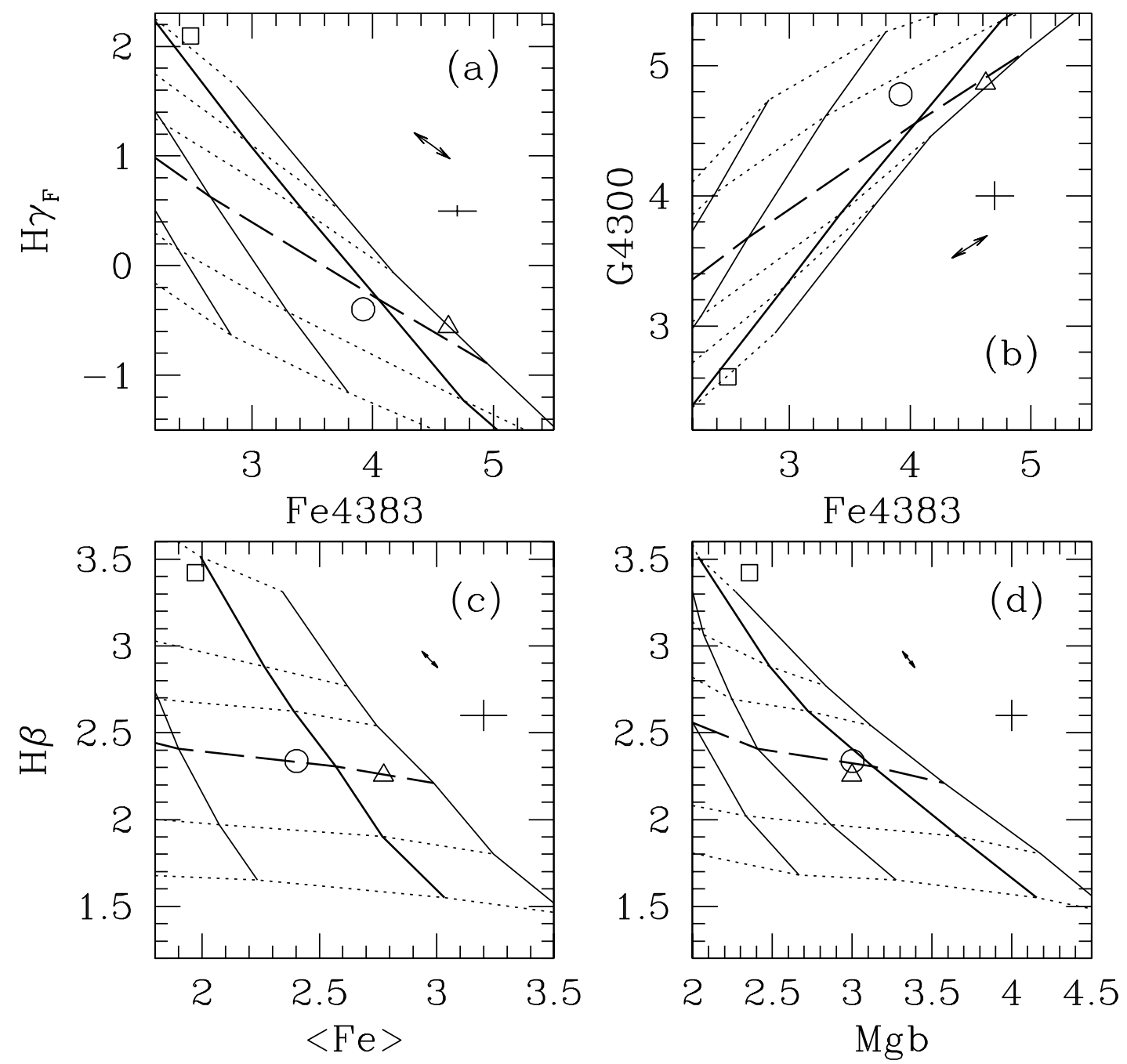

FIg. 7.- Same as Fig. 6. Symbols are the same as for Fig. 6. The most important conclusions to be drawn from this figure and Fig. 6 are (1) all Balmer lines indicate the same age for M67, in agreement with the CMD-based age; (2) the $\mathrm{Fe}, \mathrm{CN}$, and Mg indices indicate solar abundances within \pm 0.1 dex, in agreement with spectroscopic studies of stellar members; (3) when BSs are included in the integrated spectrum of M67, the spectroscopic age drops to $\sim 1.2$ Gyr, or even younger, depending on the line index adopted. For the $\mathrm{G}$ band the dependence on age is inverted, so that age decreases from top (14.1 Gyr) to bottom (2.0 Gyr). Ages based on the $\mathrm{G}$ band are too old (a factor of 2 in the case of M67). For M32, bluer Balmer lines tend to indicate younger ages, and bluer Fe lines indicate higher metallicities. The data on M32 indicate that it has supersolar iron abundance and is underabundant in magnesium relative to iron. See discussion in the text.

metal abundance indicators $(\mathrm{CN} 1, \mathrm{CN} 2, \mathrm{Fe} 4383,\langle\mathrm{Fe}\rangle$, and $\mathrm{Mg} b$ ). Index definitions can be found in Worthey et al. (1994) and Worthey \& Ottaviani (1997). In the figures, dotted lines connect models with the same age, and solid lines connect models with the same metallicity. The ages plotted are, from top to bottom, 1.2, 2.0, 2.5, 3.5, 7.9 and $14.1 \mathrm{Gyr}$, and the metallicities are, from right to left, $[\mathrm{Fe} / \mathrm{H}]=+0.2,0.0,-0.4$, -0.7 , and -1.3 (the latter two are absent in some plots, for clarity). The lines connecting models with $[\mathrm{Fe} / \mathrm{H}]=0$ and with $t=3.5 \mathrm{Gyr}$ are thicker. Indices measured in the spectra of M67 with and without BSs are represented by the open square and circle, respectively.

The most striking feature in these figures is that all Balmer lines indicate the same age for M67, within less than 1 Gyr. Moreover, the spectroscopic age according to the Balmer lines is $\sim 3.5 \mathrm{Gyr}$, in agreement with that inferred in $\S 4.1$ from the comparison of cluster data with theoretical isochrones in the CMD. We highlight the fact that the same isochrones were employed in deriving the spectroscopic and CMD ages. Even though such consistency should in principle be expected, we note that previous studies found huge discrepancies between
CMD-based and spectroscopic ages for a well-known Galactic globular cluster, 47 Tuc. The turnoff age of this cluster is $11 \pm 1 \mathrm{Gyr}$ when the isochrones of Salaris and collaborators (see description in Vazdekis et al. 2001) are employed. However, Gibson et al. (1999) found a spectroscopic age in excess of $20 \mathrm{Gyr}$ for the cluster. Later on, Vazdekis et al. (2001) showed that better agreement was reached when effects like $\alpha$-enhancement and diffusion of heavy elements are taken into account. However, a mismatch of $4 \mathrm{Gyr}$ was still lingering. Schiavon et al. (2002b) finally showed that most of the discrepancy was due to a mismatch between the theoretical luminosity function of the brighter giant stars and the observations, in the sense that theory predicted too few bright giants. Therefore, considering the previous history, it is quite reassuring that our single stellar population models provide results at this level of consistency, in a regime where SP models have never been tested before in such a level of detail.

The remaining age indicator, the $\mathrm{G}$ band (G4300), presents a trend as a function of age that is opposite to that of Balmer lines: it gets weaker for younger ages. This is due to the fact that it is much stronger in giants than in turnoff stars. As a 
result, the $G$ band gets stronger in the integrated spectra of older stellar populations because they are characterized by a higher relative contribution of giant stars to the integrated light. Being mostly due to the $\mathrm{CH}$ molecule, the $\mathrm{G}$ band is not expected to be a clean age indicator, due to its dependence on the abundance of carbon. In fact, we obtain an older age for M67 from the EW of the G band ( 6 Gyr). Tripicco \& Bell (1995) showed that the $G$ band is mostly sensitive to the abundance of carbon. According to their calculations, the mismatch between the model for $[\mathrm{Fe} / \mathrm{H}]=0.0$ and age $=3.5 \mathrm{Gyr}$ is such that it could be explained by M67 having a higher than solar $[\mathrm{C} / \mathrm{Fe}]$. However, abundance studies of M67 turnoff stars point at a different direction: they have nearly solar $[\mathrm{C} / \mathrm{Fe}]$ and may even perhaps be a little carbon-poor (see discussion in $\S 4.2 .3)$.

Therefore, it is quite puzzling that in the M67 integrated spectrum the $\mathrm{G}$ band is stronger than in the model for $3.5 \mathrm{Gyr}$ and solar metallicity. We tried removing stars very deviant from the average relation between the EW of the $\mathrm{G}$ band and color, but that did not remove the discrepancy. We showed in $\S 2.2 .1$ that the calibration of our $\mathrm{G}$ band measurements into the Lick/IDS system was somewhat uncertain. We thus believe that calibration uncertainties related to the conversion into the Lick/IDS EW system are responsible for this mismatch.

In $\S 3.1$, we corrected our model predictions for the effect of AGB stars, by adding an extra 0.1 dex in the luminosity function of the upper GB. In their analysis of the integrated spectrum of 47 Tuc, Schiavon et al. (2002b) showed that an error of \pm 0.1 dex in the luminosity function of giants brighter than the horizontal branch translates into an error of \pm 1 Gyr in the spectroscopic age inferred from Balmer lines. The latter result is valid for an old stellar population $(\sim 11 \mathrm{Gyr})$ with $[\mathrm{Fe} / \mathrm{H}]=-0.7$. For a younger and more metal-rich stellar population, we expect the effect to be less important, given that the total contribution by cool giants to the optical integrated light of metal-rich SPs with intermediate ages is lower. In fact, we note that the integrated spectrum of M67 is little affected by uncertainties in the luminosity function of the upper GB: the change in the M67 indices in Figures 6 and 7 that would be generated by a 0.1 dex variation in the luminosity function above the horizontal branch is in all cases smaller than the symbol size.

In summary, we achieve excellent consistency for M67 between the spectroscopic ages inferred from all Balmer lines studied. We attribute the mismatch with the spectroscopic age inferred from the $\mathrm{G}$ band to uncertainties in the calibration of the latter.

\subsubsection{Metal Abundances of M67 According to the SP Models}

Figures 6 and 7 also allow checking the consistency of SP model predictions with the known elemental abundances of M67. This is a unique opportunity, because M67 stars have been the subject of many abundance analyses by different groups, so that it can be fairly said that the abundance pattern of the cluster is well known. The importance of enforcing consistency between SP models and the results of classical stellar abundance analyses lies in the fact that the latter provide the only physically meaningful measurement of the abundances of heavy elements in stars. We emphasize that such a tight consistency is seldom attempted in the literature. A similar approach was followed in our study of the mildly metal-rich Galactic globular cluster, 47 Tuc (Schiavon et al. 2002a, 2002b), and now we provide, for the first time, a check of the consistency of SP models in the regime of intermediate age and solar metallicity. For this purpose, we below discuss separately a few relevant elements, together with a brief summary of the previous results from detailed abundance analysis of M67 stars.

\subsubsection{Iron}

Classical abundance analysis of M67 members, based on high-dispersion spectroscopy, was pioneered by Cohen (1980), who found $\langle[\mathrm{Fe} / \mathrm{H}]\rangle=-0.39$, from a study of four cluster giants. This result was revised by subsequent studies which found a higher iron abundance, near the solar value. Foy \& Proust (1981), analyzing two giant stars, found $\langle[\mathrm{Fe} / \mathrm{H}]\rangle-$ $0.1 \pm 0.1$. Garcia Lopez, Rebolo, \& Beckman (1988), who studied seven turnoff and one giant star found $\langle[\mathrm{Fe} / \mathrm{H}]\rangle=$ $+0.04 \pm 0.04$. Friel \& Boesgaard (1992) analyzed three cluster dwarfs and found $\langle[\mathrm{Fe} / \mathrm{H}]\rangle=+0.02 \pm 0.1$. Most recently, Tautvaisiene et al. (2000) found $\langle[\mathrm{Fe} / \mathrm{H}]\rangle=-0.03 \pm 0.15$, in an analysis of nine giant stars, and Shetrone \& Sandquist (2000) determined $\langle[\mathrm{Fe} / \mathrm{H}]\rangle=-0.05 \pm 0.05$ for four turnoff stars. In view of these previous studies, we assume that M67 has a solar iron abundance $(\langle[\mathrm{Fe} / \mathrm{H}]\rangle=0 \pm 0.1)$.

The line indices in Figures 6 and 7 that are most sensitive to the iron abundance are $\langle\mathrm{Fe}\rangle$ and $\mathrm{Fe} 4383$. Figures 6 and 7 indicate that both indices are consistent with $\langle[\mathrm{Fe} / \mathrm{H}]\rangle=0$ to within less than 0.1 dex.

\subsubsection{Carbon and Nitrogen}

The abundances of these elements are very important because they affect significantly the indices blueward of $4500 \AA$ due to the presence of thousands of $\mathrm{CN}$ and $\mathrm{CH}$ lines (for a discussion, see Schiavon et al. 2002a). The abundance of carbon also has an important effect on $\mathrm{Mg} b$ due to contamination by $\mathrm{C}_{2}$ lines (Tripicco \& Bell 1995). Brown (1987), analyzing stars at different evolutionary stages, found that the $\mathrm{C} / \mathrm{N}$ abundance ratio in M67 stars undergoes a sharp drop between $M_{V} \sim 3.5$ and $\sim 2.8$, as a result of the first dredge-up episode. Subsequent analyses are consistent with this result. Regarding carbon, analyses of dwarf stars by Friel \& Boesgaard (1992) and Shetrone \& Sandquist found $\langle[\mathrm{C} / \mathrm{Fe}]\rangle=-0.1 \pm 0.2$ and $0.0 \pm 0.1$, respectively, while Tautvaisiene et al. (2000), analyzing giant stars, found $\langle[\mathrm{C} / \mathrm{Fe}]\rangle=-0.2$. Nitrogen abundances are more uncertain, and also there are fewer determinations in the literature. Tautvaisiene et al. found that M67 giants have $\langle[\mathrm{N} / \mathrm{Fe}]\rangle=+0.2$, whereas Shetrone \& Sandquist, analyzing turnoff stars, found $\langle[\mathrm{N} / \mathrm{Fe}]\rangle=+0.1 \pm 0.2$. In summary, $[\mathrm{C} / \mathrm{N}]$ is $\sim 0.25$ dex lower in M67 giants than in dwarfs. In particular, we emphasize that the abundance ratios in M67 giants are nonsolar, whereas M67 dwarfs are consistent with a solar carbon abundance, while perhaps being slightly enhanced in nitrogen.

In Figure 6, it can be seen that our model prediction for $3.5 \mathrm{Gyr}$ and $[\mathrm{Fe} / \mathrm{H}]=0$ is in excellent agreement with the measurements of the $\mathrm{CN}$ indices in the integrated spectrum of M67, even though our models have, nominally, solarabundance ratios. This can be understood in view of the results of Gratton et al. (2000) and Carretta, Gratton, \& Sneden (2000), who showed that in field stars the first dredgeup operates a change in the surface composition of carbon and nitrogen that is very similar to what is seen in M67 and other clusters. Therefore, in the field stars that serve as the basis of our models, the run of $\mathrm{CN}$ abundances as a function of evolutionary stage is arguably similar to that of M67 stars, so that the effects due to stellar evolution in M67 stars are accounted for in the models by construction (this has first been pointed 
out by Gorgas et al. 1993). Therefore, no correction due to nonsolar-abundance ratios needs to be applied to our model predictions and M67 stars can be considered to constitute a single stellar population with solar $[\mathrm{CN} / \mathrm{Fe}]$.

\subsubsection{Magnesium}

The index in Figure 7 that is most sensitive to the abundance of magnesium is $\mathrm{Mg} b$, which is the most popular estimator of the metallicities of stellar populations of galaxies, and therefore it is very important that our predictions be consistent with the abundance analyses of M67 stars for magnesium. There is some disagreement in the literature as to the magnesium abundance of M67. Foy \& Proust found $\langle[\mathrm{Mg} / \mathrm{Fe}]\rangle=0$, Tautvaisiene et al. (2000) found $\langle[\mathrm{Mg} / \mathrm{Fe}]\rangle=$ +0.1 , and Shetrone \& Sandquist found $\langle[\mathrm{Mg} / \mathrm{Fe}]\rangle=-0.1$. In Figure 7, it can be seen that our model prediction for $\mathrm{Mg} b$ is somewhere between $[\mathrm{Mg} / \mathrm{Fe}]=-0.1$ and 0.0 , and so it is consistent with abundance analysis work. However, we stress that more work is needed in order to ascertain the magnesium abundance of M67.

In summary, we conclude that our model predictions are consistent to within \pm 0.1 dex with the known metal abundances of M67. This is reassuring, because it implies that our models predict the correct values of abundance-sensitive indices that are among the most widely used in stellar population work. We also emphasize that the predictions are consistent for indices that are sensitive to the abundances of a number of important chemical species, like iron and the light elements carbon and nitrogen, and magnesium. This also means that the models can be used to estimate luminosityweighted abundance ratios of galaxies in a way that is consistent with the state of the art of our knowledge of stellar abundances in the Galaxy.

\section{SPECTROSCOPIC AGE AND METAL ABUNDANCES OF M32}

We would like to start our discussion of the SP properties of M32 by reminding the reader that our spectrum is integrated within a square $3^{\prime \prime}$ on a side positioned on the center of the galaxy. This is important in view of the fact that previous studies have indicated the presence of a radial gradient in the spectroscopic properties of M32 (Davidge 1991; González 1993; Hardy et al. 1994; Worthey 2003).

Because of its morphology, proximity, and very high central surface brightness, M32 has become a benchmark for the study of stellar populations, and galaxy evolution in general. Spinrad \& Taylor (1971) and Faber (1973) pioneered the efforts to determine the luminosity-weighted mean metallicity of the central parts of M32 from low-resolution spectrophotometric observations, but O'Connell (1980) was the first to find that such data for the center of M32 could only be matched by requiring an intermediate-age population ( $\sim 5 \mathrm{Gyr})$ and near-solar metallicity. More recent attempts, based on more sophisticated methods and/or modeling, obtained similar results, with age ranging between 3 and $5 \mathrm{Gyr}$ and $[\mathrm{Fe} / \mathrm{H}]$ within $0.1 \mathrm{dex}$ from the solar value (e.g., Rose 1994; Jones \& Worthey 1995; Vazdekis \& Arimoto 1999; Trager et al. 2000; Worthey 2003; Caldwell et al. 2003). These results are confirmed by Figure 8 , where we compare the bluer part of the integrated spectrum of M67 that was constructed in $\S 3$ with the observed integrated spectrum of M32. Some of the most important spectral indices employed in our analysis are indicated in the figure. As can be seen, the two

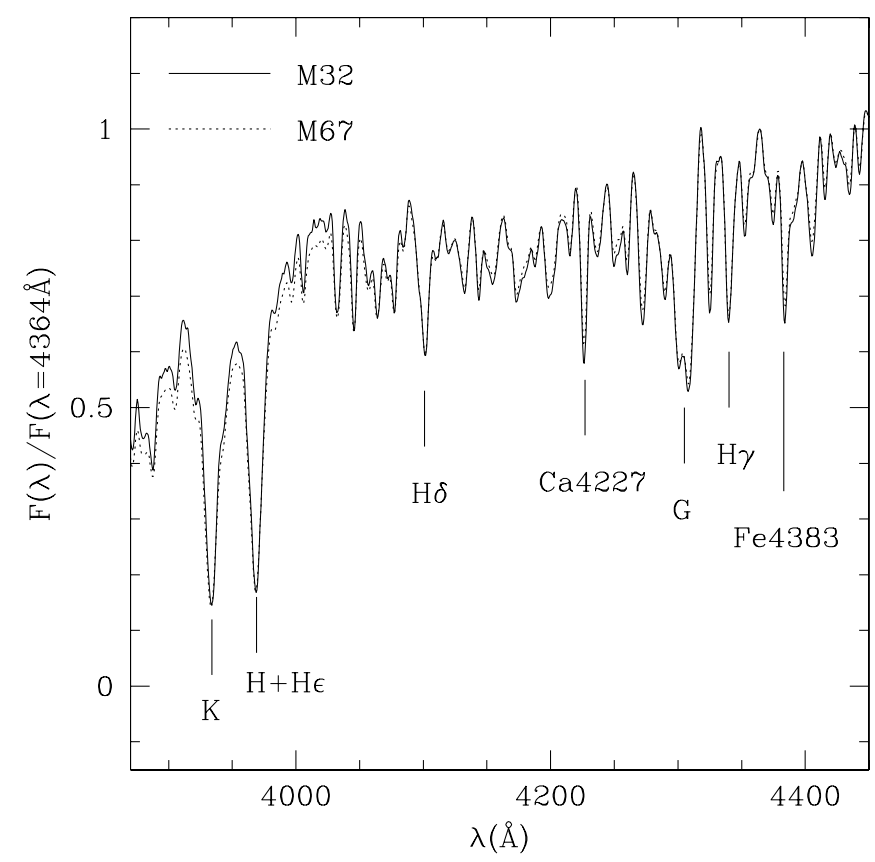

FIG. 8.-Comparison between the integrated spectra of M67 and M32. The spectra are very similar, although M32 looks to be slightly more strong-lined than M67, which suggests that M32 is on average slightly more metal-rich and younger than M67. See discussion in $\S 5$.

spectra are very similar. Actually, the integrated spectrum of M32 is in general slightly more strong-lined than that of M67, which suggests a slightly younger age and a slightly higher metallicity.

The latter result can be inspected more quantitatively in Figures 6 and 7, where Lick indices are compared with predictions for single SPs. In order to minimize abundance ratio effects, we first focus on the plots of Balmer lines against Fe indices. According to these plots, the spectroscopic age of M32 is somewhere between 2.0 and 3.5 Gyr, whereas its luminosityweighted iron abundance is supersolar, with $+0.1 \lesssim[\mathrm{Fe} / \mathrm{H}] \lesssim$ +0.3 . These numbers confirm our expectations based on inspection of the spectra of Figure 8.

It is important to bear in mind that the strength of Balmer lines is not solely governed by the temperature and brightness of turnoff stars: other families of hot stars, such as BSs and hot horizontal-branch (HB) stars can affect Balmer line strengths, thus hindering their use as age indicators (e.g., Freitas Pacheco \& Barbuy 1995; Lee, Yoon, \& Lee 2000; Maraston et al. 2003). In principle, a small population of hot stars could play a major role in strengthening the Balmer lines in M32, thereby reducing, or eliminating, the necessity for the $\sim 2-$ 3 Gyr old mean population implied by the Balmer line strengths. However, Caldwell et al. (2003) have demonstrated that to explain the enhanced Balmer lines in M32 requires a $\sim 30 \%$ contribution from a hot-star component, whether from a blue HB or BSs. On the other hand, they also show that the $\mathrm{Ca}$ II index, which levers the strength of $\mathrm{Ca}$ II $\mathrm{K}$ versus $\mathrm{H} \epsilon+\mathrm{Ca}$ II $\mathrm{H}$, restricts the contribution from hot stars to a level of $\sim 7 \%$. In addition, M32 has the weakest UV upturn in the sample of Burstein et al. (1988), so that its relative number of hot HB stars must be low. In principle, cooler BS stars, that do not make such a strong impact on the above-mentioned $\mathrm{Ca}$ II index, could be present in M32, but then they would have to exist in exceedingly large numbers, in fact rivalling the numbers of true MSTO stars. In short, we have reasons to be 
confident that our Balmer line measurements primarily provide an estimate of the spectroscopic age of M32.

With the above caveats in mind, we now focus on the plots of Balmer lines against $\mathrm{CN}$ and $\mathrm{Mg}$ indices, in order to assess the abundance ratios of M32. The $\mathrm{Mg} b$ index is mostly sensitive to magnesium. In Figures $7 c$ and $7 d$, it is seen that M32 has $[\mathrm{Fe} / \mathrm{H}] \sim+0.1$ according to $\langle\mathrm{Fe}\rangle$, while, according to $\mathrm{Mg} b$, it is about 0.1 dex below the solar-metallicity model, which is strongly suggestive of $[\mathrm{Mg} / \mathrm{Fe}]<0$. In the case of the $\mathrm{CN}$ indices (Fig. $6 a$ and $6 b$ ), for both the M32 data fall nearly on top of the $[\mathrm{Fe} / \mathrm{H}]=+0.2$ model. Since both $\mathrm{CN} 1$ and $\mathrm{CN} 2$ are mostly sensitive to the combined effect of nitrogen and carbon abundances, and given the above discussion on the iron abundance of M32, it is likely that M32 has a nearly solar abundance of carbon and/or nitrogen relative to iron.

Abundance ratios can be better assessed in plots of metal index against metal index. Such plots are shown in Figure 9, where we compare the measurements with the model predictions of single stellar populations for $[\mathrm{Fe} / \mathrm{H}]=0.0$ and +0.2 . The lines connecting models with same age are omitted in these plots for clarity. In Figure $9 b$ it can be seen that while
M67 looks like having strictly solar [Mg/Fe], M32 deviates by roughly $3 \sigma$ from the model prediction for solar-abundance ratios, which confirms the suggestion above that it has $[\mathrm{Mg} / \mathrm{Fe}]<0$. The case for nonsolar-abundance ratios for carbon and/or nitrogen is perhaps less convincing, because it depends to some extent on the $\mathrm{Fe}$ abundance indicator adopted. In Figure $9 c$, where $\mathrm{CN} 1$ is plotted against Fe4383, M32 is clearly deviating from the solar ratio models, but less so when $\mathrm{CN} 1$ is compared with $\langle\mathrm{Fe}\rangle$ in Figure $9 d$ (essentially the same results are obtained in plots involving $\mathrm{CN} 2$ ). We point out that our results agree with those of Worthey (2003) as regards $[\mathrm{Mg} / \mathrm{Fe}]$, but we are in disagreement when it comes to the abundances of carbon and nitrogen, for which he found an enhancement relative to iron of $\sim 0.2 \mathrm{dex}$.

Finally, we would like to point out an apparent systematic trend of the mean age and metallicity inferred for M32 as a function of wavelength: the resulting age looks lower, and the metallicity looks higher, as we go to bluer indices. According to the $\mathrm{H} \beta$ versus $\langle\mathrm{Fe}\rangle$ plot, the central parts of M32 have luminosity-weighted mean metallicity and age given by $[\mathrm{Fe} / \mathrm{H}] \sim$ +0.1 and $\sim 3.5$ Gyr. According to the $\mathrm{H} \gamma_{F}$ versus Fe4383 plot, $[\mathrm{Fe} / \mathrm{H}]$ is slightly above +0.2 , and the mean age is $\sim 2.5 \mathrm{Gyr}$,
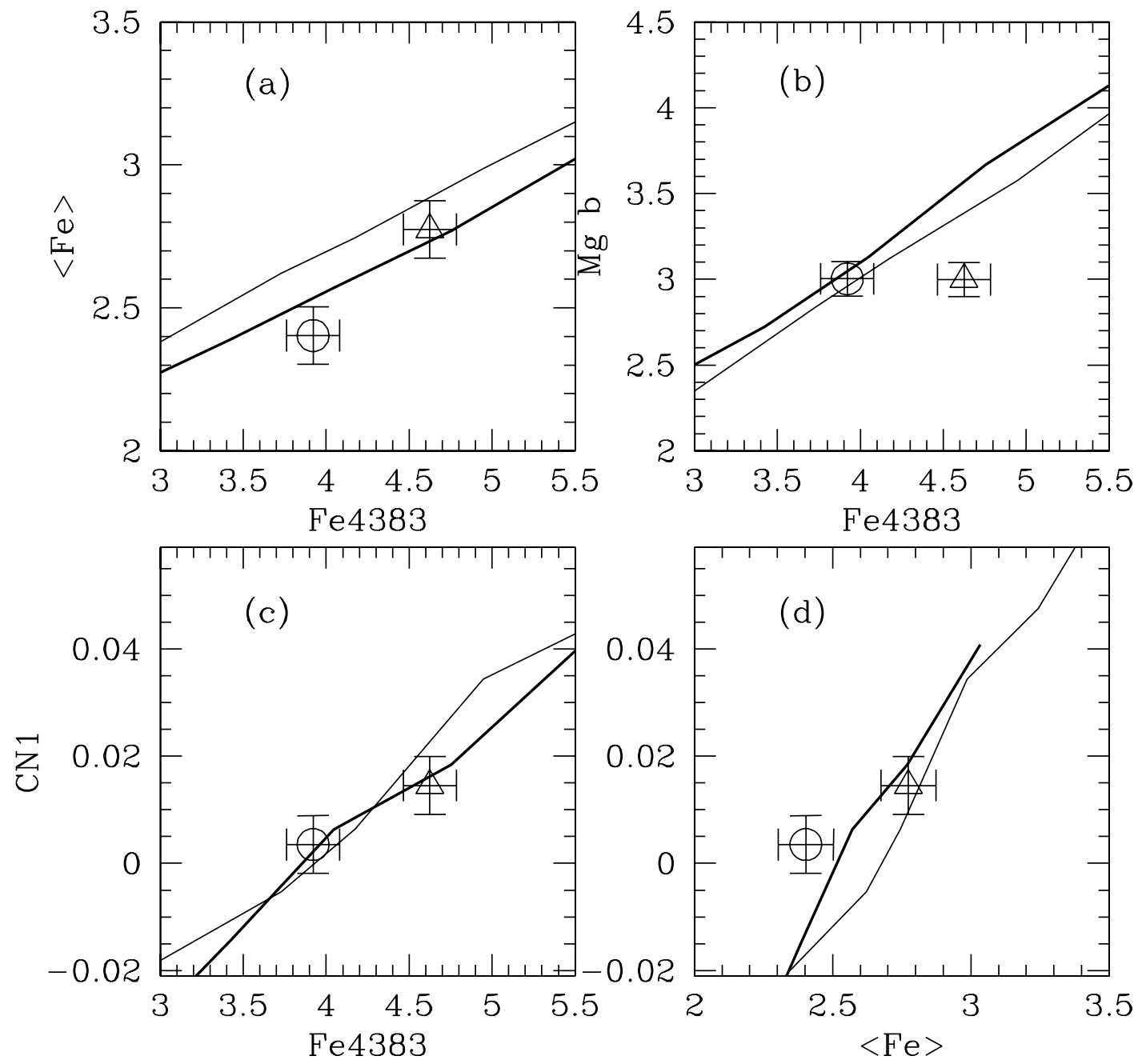

FIG. 9.-Data on M67 and M32 compared with single SP models on plots involving only abundance-sensitive indices. The symbols adopted are the same as in Fig. 6. Such plots are useful to unveil nonsolar-abundance ratios. Again, the open triangle and circle represent M67 and M32, respectively. The model lines are for $[\mathrm{Fe} / \mathrm{H}]=0.0$ and +0.2 . The former is represented by a thick line. The lines connecting same-age models are omitted for clarity. While M67 is basically consistent with solar in all abundance ratios, M32 appears to have $[\mathrm{Mg} / \mathrm{Fe}]$ substantially below solar. The combined abundances of $\mathrm{C}$ and $\mathrm{N}$ appear to be nearly solar in the center of M32. 
while in the $\mathrm{H} \delta_{F}$ versus $\mathrm{Fe} 4383$ plot the mean metallicity is even higher and the mean age younger ( 2.0 Gyr). Even though the spread in the age determinations is relatively small (because of the high age sensitivity of Balmer lines in this age regime), it is significant, given the small error bars in our measurements. If we assume that the mean age and $[\mathrm{Fe} / \mathrm{H}]$ of $\mathrm{M} 32$ are as given by the $\mathrm{H} \beta$ versus $\langle\mathrm{Fe}\rangle$ plot $(3.5 \mathrm{Gyr},+0.1 \mathrm{dex})$, then the measurements of both $\mathrm{H} \gamma_{F}$ and $\mathrm{H} \delta_{F}$ are off their predicted positions when plotted against Fe4383 by more than $2 \sigma$.

This result should be contrasted against the case of M67, for which we found essentially the same age for all Balmer lines and iron abundances according to different $\mathrm{Fe}$ indices in agreement to within 0.1 dex. M32 differs from M67 in two basic aspects: (1) it is not a single stellar population, but rather a mix of stars of different metallicities (Grillmair et al. 1996) and possibly different ages; and (2) as discussed above, M32 has an abundance pattern that differs from that of the Sun.

A simple calculation shows that a very small fraction of a young ( 1.5 Gyr) stellar population on top of an older one can reproduce data for Balmer and Fe lines. The younger stellar population is much brighter in the blue, so that it would affect bluer spectral indices more strongly with the result that the bluer Balmer lines of such a simple composite stellar population would indicate younger ages.

On the other hand, it might be also possible to reproduce the data by applying the method championed by Trager et al. (2000), where the absorption line indices are corrected for varying abundance ratios, based on the computations of synthetic stellar spectra by Trippico \& Bell (1995). The latter does not include calculations for $\mathrm{H} \delta_{F}$ and $\mathrm{H} \gamma_{F}$ though, because these indices were defined later, by Worthey \& Ottaviani (1997). This is important because we have shown, in Schiavon et al. (2002a), that both $\mathrm{H} \gamma_{F}$ and $\mathrm{H} \delta_{F}$ are influenced by variations in the abundances of carbon and nitrogen, due to the contamination by $\mathrm{CN}$ and $\mathrm{CH}$ lines. While we cannot correct the higher order Balmer lines for the effects of unknown abundance ratios, we recognize that they might be at least partially responsible for the results of Figures 6 and 7.

It is very hard to disentangle the effect of varying abundance ratios from the potential age mix of stellar populations. As a consequence, when blue indices are involved, it is difficult to apply the method devised by Trager et al. (2000) to assess nonsolar-abundance ratios. The reason is that the method essentially seeks the combination of metal abundances that brings the ages and metallicities into agreement according to the different line indices, so that it tends to wash out any effect due to a mix of stellar populations of different ages. In a future paper, we will address this problem in more detail.

\section{CONCLUSIONS}

We have constructed an integrated spectrum of the Galactic cluster M67, from spectroscopic observations of bona fide cluster members. The resulting integrated spectrum was used as a template for stellar population synthesis, through comparison with model predictions for single stellar populations. We contrasted the spectroscopic age and metal abundances estimated from the SP models with the age obtained from the cluster CMD and elemental abundances determined in previous works from high-dispersion abundance analyses. It is the first time that such models are tested to this level of precision in the solar-metallicity, intermediate-age regime. Our SP models were then applied to the study of the integrated spectrum of M32, yielding reliable determinations of the galaxy's light-weighted mean age and metal abundances. We summarize below our main conclusions.

1. Our models for the known age and metal abundances of M67 match very well its integrated spectrum. Adopting the Girardi et al. (2000) theoretical isochrones for solar-metallicity and solar-abundance ratios, we matched both the colormagnitude data and integrated spectrum of M67, for an age of 3.5 Gyr and solar metallicity, to within $0.5 \mathrm{Gyr}$ and $0.1 \mathrm{dex}$, respectively. Most importantly, we found very good consistency for the ages derived from $\mathrm{H} \beta, \mathrm{H} \gamma$, and $\mathrm{H} \delta$. This result demonstrates the remarkable degree of consistency of our models. We also performed a detailed check to see whether our model predictions are consistent with the known elemental abundances of M67 stars. We found that the predictions from SP models for the abundances of iron, carbon, nitrogen, and magnesium agree with the elemental abundance analyses of individual stars to within 0.1 dex.

2. The integrated spectrum of M32 is very similar to, although slightly more strong-lined than, that of M67. On the basis of our newly tested models, we perform an analysis of the integrated spectrum of M32, with the aim of estimating its light-weighted mean age and metal abundances. We found that the spectroscopic age of M32 is somewhere between 2 and 3.5 Gyr, depending on the Balmer line adopted. For the mean metal abundances, we found $[\mathrm{Fe} / \mathrm{H}]$ ranging between +0.1 and +0.4 , again depending on the metallicity indicators adopted. For light elements, we found M32 to be underabundant in magnesium relative to iron, with $[\mathrm{Mg} / \mathrm{Fe}] \sim-0.2$, whereas the combined abundances of carbon and nitrogen seem to be nearly solar relative to iron. A more in-depth study of the composite nature of the stellar populations in M32 is deferred to a forthcoming paper.

3. Contrary to the case of M67, application of our single stellar population models to the integrated spectrum of the central parts of M32 revealed a systematic effect in the resulting ages and metallicities as a function of wavelength. Use of red indices results in a mean age $\sim 1.5$ times older and a metallicity $\sim 0.3$ dex lower than when blue indices are adopted. This result can be due to either the effects of nonsolar-abundance ratios on blue absorption indices and/or to a mix of stellar populations in M32 with different ages.

4. In agreement with previous studies, we found that BSs contribute with an unusually high fraction of the blue integrated light of M67. This result is robust, since our integrated spectrum is constructed on the basis of photometry that is complete down to many magnitudes below the turnoff. We suggest that this result might be due to the cluster's past history of severe mass segregation, followed by evaporation of low-mass stars.

Calibrating models by comparison with integrated spectra of clusters has become a standard procedure in the field of stellar population synthesis. The nature of the Galactic globular cluster system, and the limitations of our knowledge of the detailed abundance pattern of their constituent stars, has prevented an accurate calibration of such models with the degree of accuracy that is required for many of their applications. The latter is particularly true in the regime of solar metallicity and intermediate ages, which is of great importance in view of its potential application for the study of stellar populations of distant systems, for which the look-back times are more than half the Hubble time. This work is an attempt at filling this gap. Applications of our models to the study of 
galaxies both nearby and at cosmological distances are currently under way.

We would like to thank S. Faber for enlightening discussions during the course of this investigation. The referee, Guy Worthey, is thanked for comments and suggestions that greatly improved this paper. We also thank Hyun-chul Lee for a careful reading of an earlier version of this manuscript.
R. P. S. acknowledges support provided by the National Science Foundation (NSF) through grant GF-1002-99 and from the Association of Universities for Research in Astronomy, Inc., under NSF cooperative agreement AST 96-13615 (Gemini Fellowship). Support from the NSF, through grant AST 00-71198 to the University of California, Santa Cruz, and from $\mathrm{CNPq} / \mathrm{Brazil}$, under grant 200510/99-1, are also deeply appreciated. J. A. R. acknowledges support from NSF grant AST 99-00720 to the University of North Carolina.

\section{APPENDIX}

\section{LICK/IDS INDEX MEASUREMENTS}

Table 6 contains Lick/IDS index measurements taken in the spectra of all the field and M67 stars observed in this work.

TABLE 6

Lick Indices Measured in the Spectra of Field and M67 Stars

\begin{tabular}{|c|c|c|c|c|c|c|c|c|c|c|c|}
\hline ID & $\mathrm{H} \delta_{F}$ & $\mathrm{CN} 1$ & $\mathrm{CN} 2$ & G4300 & $\mathrm{H} \gamma_{F}$ & $\mathrm{Fe} 4383$ & $\mathrm{H} \beta$ & $\operatorname{Mg} b$ & $\mathrm{Mg}_{2}$ & Fe5270 & $\mathrm{Fe} 5335$ \\
\hline HD $37216 \ldots \ldots$ & -0.0927 & -0.0268 & -0.0090 & 5.5800 & -1.7390 & 5.0640 & 1.9310 & 4.0450 & 0.1866 & 2.6840 & 2.3580 \\
\hline HD $47914 \ldots \ldots$ & -1.3580 & 0.1929 & 0.2470 & 6.0950 & -3.6050 & 8.9290 & 0.6220 & 4.6620 & 0.4279 & 4.3080 & 4.3690 \\
\hline HD $49178 \ldots \ldots$ & 0.7858 & -0.0070 & 0.0108 & 5.2630 & -0.6413 & 3.7520 & 2.6100 & 3.2390 & 0.1454 & 2.1010 & 1.8940 \\
\hline HD $60522 \ldots \ldots$ & -1.2280 & 0.1305 & 0.1837 & 5.8620 & -3.2510 & 8.7260 & 0.7104 & 4.7330 & 0.4284 & 4.2310 & 4.3430 \\
\hline HD 61606A.... & -1.1130 & 0.0038 & 0.0308 & 5.7230 & -3.2210 & 7.5730 & 0.9142 & 5.9370 & 0.3588 & 3.7920 & 3.6200 \\
\hline HD $62721 \ldots \ldots$ & -0.9038 & 0.1198 & 0.1765 & 6.0490 & -3.0220 & 7.7080 & 0.4400 & 4.8540 & 0.4132 & 3.8200 & 3.7120 \\
\hline HD $69582 \ldots \ldots$ & 0.4846 & -0.0427 & -0.0294 & 4.9650 & -0.6286 & 4.0670 & 2.4240 & 3.3490 & 0.1475 & 2.4380 & 2.0690 \\
\hline HD $69830 \ldots \ldots$ & -0.0913 & -0.0208 & -0.0053 & 5.7350 & -1.8680 & 4.9510 & 1.9740 & 4.3250 & 0.1949 & 2.6830 & 2.2900 \\
\hline HD $70272 \ldots \ldots$ & -1.4280 & 0.1288 & 0.1807 & 5.8610 & -3.0130 & 8.8740 & 0.7205 & 4.2880 & 0.3889 & 4.3060 & 4.3320 \\
\hline HD $94705 \ldots \ldots$ & -0.6903 & -0.1174 & -0.0824 & 3.5000 & -2.2890 & -0.2740 & 5.0380 & 14.0800 & 0.4313 & 3.4070 & 1.2200 \\
\hline HD $98991 \ldots \ldots$ & 3.3470 & -0.1002 & -0.0778 & 1.5110 & 3.5730 & 0.8280 & 4.3510 & 0.8016 & 0.0480 & 1.1230 & 0.9891 \\
\hline HD $106156 \ldots$ & -0.1425 & 0.0264 & 0.0430 & 5.6730 & -1.8050 & 5.4120 & 2.1650 & 4.4290 & 0.2197 & 3.0360 & 2.6080 \\
\hline HD $128987 \ldots$. & 0.1842 & -0.0374 & -0.0236 & 4.9530 & -1.0410 & 4.3020 & 2.1810 & 3.7900 & 0.1631 & 2.6530 & 2.2100 \\
\hline HD $126511 \ldots .$. & 0.0126 & 0.0123 & 0.0278 & 5.4960 & -1.6630 & 4.9920 & 2.3380 & 4.2010 & 0.1986 & 2.5700 & 2.4120 \\
\hline HD $136834 \ldots$. & -1.1980 & 0.1275 & 0.1618 & 5.9300 & -3.5640 & 7.9070 & 1.2520 & 6.4510 & 0.4054 & 4.1830 & 3.8950 \\
\hline HD $158614 \ldots$. & 0.4254 & -0.0222 & -0.0110 & 5.3790 & -1.1710 & 4.0860 & 2.4000 & 3.5730 & 0.1599 & 2.4140 & 2.1240 \\
\hline HD $165341 \ldots$. & -0.4514 & 0.0045 & 0.0196 & 5.6020 & -2.2010 & 5.5210 & 1.7720 & 4.7960 & 0.2407 & 3.2220 & 2.7730 \\
\hline $5041 \ldots \ldots \ldots \ldots \ldots$ & 1.8350 & -0.0712 & -0.0505 & 4.1610 & 1.1570 & 2.0860 & 3.1420 & 1.4940 & 0.0735 & 1.7170 & 1.3170 \\
\hline $5059 \ldots \ldots \ldots \ldots \ldots$ & -0.6574 & 0.0772 & 0.0985 & 6.4420 & -2.7460 & 5.4150 & 1.6330 & 3.4310 & 0.2000 & 2.9500 & 2.4030 \\
\hline $5118 \ldots \ldots \ldots \ldots \ldots$ & 2.5360 & -0.0914 & -0.0688 & 2.9890 & 2.2230 & 1.2170 & 3.5770 & 1.3260 & 0.0758 & 1.3320 & 1.0400 \\
\hline $5169 \ldots \ldots \ldots \ldots \ldots$ & 2.1560 & -0.0833 & -0.0597 & 3.6500 & 1.6450 & 1.8200 & 3.4600 & 1.5350 & 0.0795 & 1.5380 & 1.3140 \\
\hline $5191 \ldots \ldots \ldots \ldots \ldots$ & 3.0040 & -0.1002 & -0.0786 & 2.2490 & 2.8480 & 1.0120 & 3.8600 & 1.2530 & 0.0623 & 1.3220 & 1.1000 \\
\hline $5228 \ldots \ldots \ldots \ldots$ & -0.1325 & 0.0209 & 0.0363 & 6.2920 & -2.1600 & 4.8060 & 1.7300 & 3.0820 & 0.1529 & 2.5730 & 2.0500 \\
\hline $5248 \ldots \ldots \ldots \ldots \ldots$ & 2.4470 & -0.0860 & -0.0652 & 3.5480 & 1.7650 & 1.3220 & 3.3970 & 1.3930 & 0.0838 & 1.4070 & 1.1990 \\
\hline $5249 \ldots \ldots \ldots \ldots$ & 2.2360 & -0.0822 & -0.0638 & 3.7910 & 1.4580 & 2.0730 & 3.3650 & 1.8860 & 0.0899 & 1.6140 & 1.3370 \\
\hline $5284 \ldots \ldots \ldots \ldots \ldots$ & 2.4350 & -0.0896 & -0.0691 & 3.3520 & 1.9440 & 1.5680 & 3.5410 & 1.2500 & 0.0718 & 1.4170 & 1.1730 \\
\hline $5318 \ldots \ldots \ldots \ldots \ldots$ & -0.5367 & 0.0583 & 0.0783 & 6.2910 & -2.6440 & 5.7050 & 1.6200 & 3.6720 & 0.2010 & 3.0350 & 2.5910 \\
\hline $5342 \ldots \ldots \ldots \ldots \ldots$ & 2.4070 & -0.0882 & -0.0683 & 3.8590 & 1.5780 & 1.7010 & 3.3740 & 1.5190 & 0.0850 & 1.4940 & 1.1640 \\
\hline $5350 \ldots \ldots \ldots \ldots \ldots$ & 0.3455 & -0.0233 & -0.0069 & 5.8260 & -1.2980 & 4.5590 & 2.3520 & 3.0070 & 0.1 & & 2.2000 \\
\hline $5362 \ldots \ldots \ldots \ldots \ldots$ & 0.7275 & -0.0467 & -0.0328 & 5.6220 & -0.7988 & 3.3720 & 2.3280 & 2.1620 & 0.1239 & 2.0650 & 1.6160 \\
\hline $5451 \ldots \ldots \ldots \ldots \ldots$ & 1.5520 & -0.0627 & -0.0474 & 3.5540 & 0.7303 & 2.4130 & 2.2350 & 1.9260 & 0.1018 & 1.8950 & 1.4650 \\
\hline $5544 \ldots \ldots \ldots \ldots \ldots$ & 1.4190 & -0.0590 & -0.0376 & 4.8810 & 0.2851 & 2.5190 & 2.6930 & 2.0140 & 0.1047 & 1.7630 & 1.5410 \\
\hline $5571 \ldots \ldots \ldots \ldots \ldots$ & 2.9790 & -0.1046 & -0.0779 & 2.5040 & 2.9570 & 1.0840 & 4.1210 & 0.9612 & 0.0517 & 1.3190 & 1.1960 \\
\hline $5583 \ldots \ldots \ldots \ldots \ldots$ & 2.2060 & -0.0825 & -0.0601 & 3.6910 & 1.6270 & 1.7870 & 3.3220 & 1.6920 & 0.0722 & 1.5700 & 1.2890 \\
\hline $5586 \ldots \ldots \ldots \ldots$ & 2.4330 & -0.0931 & -0.0688 & 3.1840 & 2.1150 & 1.9050 & 3.5070 & 1.2550 & 0.0708 & 1.4060 & 1.2950 \\
\hline $5643 \ldots \ldots \ldots \ldots \ldots$ & 0.8413 & -0.0290 & -0.0105 & 4.7740 & -0.2366 & 3.3870 & 2.2390 & 2.5120 & 0.1412 & 2.3450 & 2.0180 \\
\hline $5667 \ldots \ldots \ldots \ldots \ldots$ & 3.8030 & -0.1166 & -0.0924 & 1.1210 & 3.8120 & 0.6684 & 4.6040 & 1.1990 & 0.0626 & 1.1680 & 1.0500 \\
\hline $5679 \ldots \ldots \ldots \ldots$ & 2.3330 & -0.0872 & -0.0662 & 3.7270 & 1.7080 & 1.8900 & 3.4330 & 1.4100 & 0.0767 & 1.3710 & 1.2590 \\
\hline $5688 \ldots \ldots \ldots \ldots \ldots$ & 2.3600 & -0.0883 & -0.0635 & 3.2900 & 1.9500 & 1.7650 & 3.5380 & 1.5120 & 0.0789 & 1.4120 & 1.3480 \\
\hline 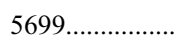 & 2.1560 & -0.0762 & -0.0557 & 3.6260 & 1.5890 & 1.8640 & 3.4010 & 1.5540 & 0.0812 & 1.4880 & 1.3660 \\
\hline $5716 \ldots \ldots \ldots \ldots \ldots$ & 3.7310 & -0.1166 & -0.0910 & 1.4190 & 3.8170 & 0.6320 & 4.5290 & 1.1250 & 0.0577 & 1.2450 & 1.1110 \\
\hline $5741 \ldots \ldots \ldots \ldots \ldots$ & 3.5250 & -0.1098 & -0.0828 & 1.6800 & 3.3810 & 0.4970 & 4.3640 & 1.2770 & 0.0729 & 1.3030 & 1.0070 \\
\hline $5756 \ldots \ldots \ldots \ldots \ldots$ & 0.4934 & -0.0192 & -0.0015 & 5.8670 & -1.3880 & 3.9920 & 2.2090 & 2.4950 & 0.1438 & 2.2780 & 2.1380 \\
\hline $5790 \ldots \ldots \ldots \ldots \ldots$ & 1.9560 & -0.0751 & -0.0563 & 3.8440 & 1.4240 & 2.0180 & 3.2710 & 1.5170 & 0.0716 & 1.6810 & 1.3090 \\
\hline $5795 \ldots \ldots \ldots \ldots$ & 2.3000 & -0.0880 & -0.0638 & 3.8310 & 1.3350 & 1.8720 & 3.3840 & 1.7530 & 0.0916 & 1.6410 & 1.3410 \\
\hline $5833 \ldots \ldots \ldots \ldots \ldots$ & 3.5450 & -0.1085 & -0.0840 & 0.6459 & 3.8750 & 0.5400 & 4.3300 & 1.1480 & 0.0648 & 1.0540 & 1.0460 \\
\hline
\end{tabular}


TABLE 6-Continued

\begin{tabular}{|c|c|c|c|c|c|c|c|c|c|c|c|}
\hline ID & $\mathrm{H} \delta_{F}$ & $\mathrm{CN} 1$ & $\mathrm{CN} 2$ & G4300 & $\mathrm{H} \gamma_{F}$ & $\mathrm{Fe} 4383$ & $\mathrm{H} \beta$ & $\operatorname{Mg} b$ & $\mathrm{Mg}_{2}$ & Fe5270 & Fe5335 \\
\hline $5853 \ldots$ & 1.3380 & -0.0647 & -0.0478 & 5.0660 & 0.1488 & 2.6760 & 2.8280 & 1.6980 & 0.0841 & 1.8720 & 1.2700 \\
\hline $5855 \ldots$ & -0.5064 & 0.0567 & 0.0814 & 6.2290 & -2.5850 & 5.4600 & 1.5810 & 3.4320 & 0.1819 & 2.7090 & 2.4010 \\
\hline $5927 \ldots$ & -0.1161 & -0.0139 & 0.0042 & 6.1910 & -1.8380 & 4.0610 & 2.0470 & 3.0560 & 0.1669 & 2.6250 & 2.1500 \\
\hline $5929 \ldots \ldots \ldots \ldots$ & 1.8280 & -0.0715 & -0.0521 & 4.3900 & 1.0160 & 2.2430 & 3.1220 & 1.4430 & 0.0758 & 1.6710 & 1.3540 \\
\hline $5969 \ldots$ & 2.7860 & -0.0898 & -0.0680 & 2.8390 & 2.4270 & 1.2450 & 3.6860 & 1.2620 & 0.0506 & 1.4190 & 1.2740 \\
\hline $5993 \ldots$. & 1.1720 & -0.0494 & -0.0339 & 4.8060 & -0.0446 & 2.7430 & 2.6520 & 2.0150 & 0.1135 & 1.8920 & 1.4980 \\
\hline $5996 \ldots$ & 0.6355 & -0.0346 & -0.0239 & 5.5530 & -0.7603 & 3.6020 & 2.4280 & 2.2500 & 0.1211 & 2.0740 & 1.7290 \\
\hline $5997 \ldots$ & -0.7729 & 0.1205 & 0.1478 & 6.2870 & -2.6590 & 5.7530 & 1.5230 & 3.3980 & 0.1942 & 2.9150 & 2.5600 \\
\hline $6089 \ldots$ & 2.0870 & -0.0813 & -0.0615 & 3.8510 & 1.4750 & 2.0260 & 3.2100 & 1.5350 & 0.0633 & 1.6480 & 1.3260 \\
\hline $6107 \ldots \ldots \ldots \ldots$ & 0.6495 & -0.0394 & -0.0272 & 5.2630 & -0.7022 & 3.2150 & 2.4020 & 2.1970 & 0.1223 & 2.0390 & 1.7040 \\
\hline 6114.................... & -0.6396 & 0.0471 & 0.0607 & 6.3370 & -2.4530 & 5.5260 & 1.7060 & 3.3890 & 0.1808 & 2.7740 & 2.3590 \\
\hline $6134 \ldots$ & 2.2270 & -0.0872 & -0.0637 & 3.5200 & 1.5980 & 1.8430 & 3.4880 & 1.5910 & 0.0891 & 1.5020 & 1.2290 \\
\hline $6158 \ldots$ & 1.3580 & -0.0649 & -0.0489 & 4.5780 & 0.6104 & 2.6430 & 2.6800 & 1.9630 & 0.0940 & 1.7930 & 1.5690 \\
\hline $6169 .$. & -0.5138 & 0.0632 & 0.0806 & 6.0210 & -2.5500 & 5.3930 & 1.5990 & 3.3860 & 0.1934 & 2.8360 & 2.5970 \\
\hline $6228 \ldots$ & 2.0570 & -0.0855 & -0.0689 & 3.9570 & 1.3130 & 1.9620 & 3.2580 & 1.3770 & 0.0657 & 1.5600 & 1.2440 \\
\hline $6259 \ldots \ldots \ldots$ & -0.9166 & 0.0757 & 0.1007 & 6.4420 & -2.8500 & 5.6510 & 1.5730 & 4.0570 & 0.2143 & 3.0730 & 2.4050 \\
\hline $6313 \ldots \ldots \ldots \ldots . . . . .$. & 2.2710 & -0.0868 & -0.0654 & 3.3920 & 1.8730 & 1.7610 & 3.5000 & 1.4410 & 0.0748 & 1.5120 & 1.1330 \\
\hline $6395 \ldots \ldots \ldots$. & 1.9910 & -0.0806 & -0.0597 & 4.2720 & 1.0910 & 2.0080 & 3.0960 & 1.9860 & 0.0891 & 1.5930 & 1.3650 \\
\hline $6408 \ldots$ & 0.2703 & -0.0144 & 0.0028 & 5.7480 & -1.2760 & 4.2950 & 2.0830 & 2.8160 & 0.1345 & 2.3000 & 1.9840 \\
\hline $6470 \ldots$ & -1.4870 & 0.2358 & 0.2844 & 6.2960 & -3.4310 & 7.7730 & 0.9060 & 3.6800 & 0.2921 & 3.8690 & 3.4910 \\
\hline $6471 \ldots$ & -1.1870 & 0.1540 & 0.2084 & 5.8500 & -3.2070 & 8.6350 & 0.7761 & 4.3940 & 0.4100 & 4.1820 & 4.3690 \\
\hline $6472 .$. & -1.1900 & 0.1644 & 0.1912 & 6.7930 & -2.8270 & 5.3920 & 1.4300 & 2.6690 & 0.1739 & 3.0650 & 2.5640 \\
\hline $6477 \ldots$ & 2.1780 & -0.0835 & -0.0606 & 3.7970 & 1.4510 & 1.8820 & 3.3550 & 1.5290 & 0.0851 & 1.6310 & 1.3730 \\
\hline $6480 \ldots \ldots$ & 4.0450 & -0.1177 & -0.0893 & 0.8838 & 4.3800 & 0.8779 & 4.8870 & 0.7455 & 0.0436 & 1.2260 & 1.0270 \\
\hline 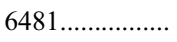 & 7.0520 & -0.2051 & -0.1683 & -2.9240 & 7.1090 & -0.6073 & 7.1850 & 0.0866 & 0.0205 & 0.0389 & 0.0213 \\
\hline $6482 .$. & -1.3470 & 0.2099 & 0.2611 & 6.2420 & -3.3350 & 7.7600 & 0.8280 & 3.8330 & 0.3172 & 3.9210 & 3.5610 \\
\hline 6484................... & 3.9380 & -0.1190 & -0.0912 & 0.4985 & 4.2160 & 0.3073 & 4.6610 & 0.8662 & 0.0595 & 1.0440 & 0.8099 \\
\hline $6485 \ldots$ & -1.2040 & 0.2296 & 0.2623 & 6.7130 & -2.9230 & 6.0340 & 1.5110 & 2.8570 & 0.1915 & 3.3000 & 2.8240 \\
\hline $6486 \ldots \ldots \ldots \ldots . . . . . . .$. & -1.3900 & 0.2418 & 0.2834 & 6.3810 & -3.4920 & 7.4740 & 1.0910 & 3.6520 & 0.2707 & 3.7370 & 3.3920 \\
\hline $6488 \ldots \ldots \ldots \ldots \ldots$ & -0.3128 & 0.0549 & 0.0778 & 5.8770 & -1.7400 & 4.6280 & 1.8610 & 2.7210 & 0.1600 & 2.5440 & 2.2240 \\
\hline 6489.................. & -1.2280 & 0.1916 & 0.2265 & 6.5360 & -2.8870 & 6.0840 & 1.3880 & 3.3180 & 0.1968 & 3.2150 & 2.6390 \\
\hline $6490 \ldots$ & 9.0460 & -0.3158 & -0.2714 & -3.7640 & 9.2760 & -1.8830 & 9.0600 & 0.4973 & 0.0164 & 0.2899 & 0.0941 \\
\hline 6491.................. & 1.5270 & -0.0628 & -0.0464 & 4.1230 & 1.0880 & 2.3480 & 3.0050 & 1.2600 & 0.0634 & 1.6230 & 1.2550 \\
\hline $6492 \ldots \ldots \ldots \ldots \ldots$ & -1.3510 & 0.2239 & 0.2554 & 6.7100 & -2.9540 & 6.2510 & 1.5190 & 2.8350 & 0.1745 & 3.2140 & 2.5730 \\
\hline 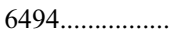 & -1.0970 & 0.2244 & 0.2599 & 6.6090 & -2.8540 & 5.7160 & 1.5210 & 2.7620 & 0.1688 & 3.1190 & 2.6600 \\
\hline $6495 \ldots \ldots \ldots \ldots \ldots$ & -1.1850 & 0.1990 & 0.2486 & 6.1260 & -3.4330 & 8.3990 & 0.6640 & 3.8390 & 0.3584 & 4.1590 & 4.1790 \\
\hline 6497.................... & -1.3540 & 0.2750 & 0.3102 & 6.0460 & -2.6810 & 6.5930 & 1.4390 & 3.3610 & 0.1985 & 3.4100 & 2.9380 \\
\hline 6499 .................. & -1.3530 & 0.2355 & 0.2879 & 6.4090 & -3.3460 & 7.8350 & 0.9870 & 3.5970 & 0.2929 & 3.8970 & 3.4580 \\
\hline $6501 \ldots \ldots \ldots \ldots \ldots$ & 7.3830 & -0.2491 & -0.2039 & -2.1570 & 8.0400 & -1.3630 & 7.9410 & 0.4634 & 0.0290 & 0.8123 & 0.5647 \\
\hline $6502 \ldots \ldots \ldots \ldots \ldots$ & -1.2750 & 0.1865 & 0.2135 & 6.6060 & -3.0200 & 6.3420 & 1.4180 & 3.6170 & 0.2331 & 3.2620 & 2.7790 \\
\hline 6503 .................... & -1.4410 & 0.2374 & 0.2736 & 6.7290 & -2.9620 & 6.1870 & 1.4620 & 2.9450 & 0.1806 & 3.2220 & 2.6680 \\
\hline $6505 \ldots \ldots \ldots \ldots \ldots$ & -1.1990 & 0.1870 & 0.2184 & 6.3550 & -2.8850 & 6.4050 & 1.4870 & 3.2760 & 0.2101 & 3.3190 & 2.7220 \\
\hline $6506 \ldots \ldots \ldots \ldots \ldots . . . . .$. & -1.3100 & 0.2188 & 0.2536 & 6.5560 & -2.8660 & 5.9900 & 1.5470 & 2.8710 & 0.1701 & 3.1120 & 2.5860 \\
\hline 6510...................... & 8.9160 & -0.3072 & -0.2640 & -3.4490 & 9.0830 & -1.8710 & 8.8540 & 0.6110 & 0.0198 & 0.3626 & 0.2005 \\
\hline $6512 \ldots \ldots \ldots \ldots \ldots$ & -1.3750 & 0.2205 & 0.2540 & 6.5910 & -2.9480 & 5.7710 & 1.5090 & 2.7030 & 0.1632 & 3.2150 & 2.6940 \\
\hline $6513 \ldots \ldots \ldots \ldots \ldots$ & -0.9952 & 0.1809 & 0.2239 & 6.4960 & -3.0620 & 6.3080 & 1.0570 & 3.2110 & 0.2281 & 3.3220 & 2.8270 \\
\hline 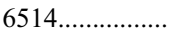 & -0.9137 & 0.0462 & 0.0918 & 5.0840 & -2.8110 & 7.7780 & 1.3350 & 6.1520 & 0.4777 & 3.9160 & 3.9860 \\
\hline $6515 \ldots \ldots \ldots \ldots \ldots$ & -1.4070 & 0.2461 & 0.2927 & 6.3500 & -3.4810 & 7.5920 & 1.0580 & 3.8170 & 0.2846 & 3.8250 & 3.4950 \\
\hline $6516 \ldots \ldots \ldots \ldots \ldots . .$. & -1.3610 & 0.2350 & 0.2659 & 6.6360 & -3.0710 & 5.9680 & 1.5370 & 2.8610 & 0.1869 & 3.2990 & 2.7320 \\
\hline Errors............ & 0.03 & 0.007 & 0.0004 & 0.005 & 0.02 & 0.04 & 0.04 & 0.08 & 0.005 & 0.02 & 0.04 \\
\hline
\end{tabular}

Nоте.-The identification numbers of M67 stars are from MMJ. The last row lists mean errors estimated as described in $\S 2.2 .1$.

Ahumada, J., \& Lapasset, E. 1995, A\&AS, 109, 375

Alonso, A., Arribas, S., \& Martínez-Roger, C. 1995, A\&A, 297, 197 1999, A\&AS, 140, 261

Balogh, M. L., Morris, S. L., Yee, H. K. C., Carlberg, R. G., \& Ellingson, E. 1999, ApJ, 527, 54

Beasley, M. A., Hoyle, F., \& Sharples, R. M. 2002, MNRAS, 336, 168 Brown, J. A. 1987, ApJ, 317, 701

Burstein, D., Bertola, F., Buson, L. M., Faber, S. M., \& Lauer, T. R. 1988, ApJ, 328,440

Carney, B. W., Latham, D. W., \& Laird, J. B. 1989, AJ, 97, 423

Carretta, E., Gratton, R. G., \& Sneden, C. 2000, A\&A, 356, 238

Caldwell, N., Rose, J. A., \& Concannon, K. D. 2003, AJ, 125, 2891

\section{REFERENCES}

Chaboyer, B., Green, E. M., \& Liebert, J. 1999, AJ, 117, 1360

Cohen, J. G. 1980, ApJ, 241, 981

Colless, M., et al. 2001, MNRAS, 328, 1039

Davidge, T. J. 1991, AJ, 101, 884

Davis, M., et al. 2003, Proc. SPIE, 4834, 161

de Freitas Pacheco, J. A., \& Barbuy, B. 1995, A\&A, 302, 718

Deng, L., Chen, R., Liu, X. S., \& Chen, J. S. 1999, ApJ, 524, 824

Faber, S. M. 1973, ApJ, 179, 731

Fabricant, D., Cheimets, P., Caldwell, N., \& Geary, J. 1998, PASP, 110, 79

Fan, X., et al. 1996, AJ, 112, 628

Fisher, D., Fabricant, D., Franx, M., \& van Dokkum, P. 1998, ApJ, 498, 195

Foy, R., \& Proust, D. 1981, A\&A, 99, 221 
Friel, E. D., \& Boesgaard, A. M. 1992, ApJ, 387, 170

Garcia Lopez, R. J., Rebolo, R., \& Beckman, J. E. 1988, PASP, 100, 1489

Gibson, B. K., Madgwick, D. S., Jones, L. A., Da Costa, G. S., \& Norris, J. E. 1999, AJ, 118, 1268

Gilmore, G., Wyse, R. F. G., \& Jones, J. B. 1995, AJ, 109, 1095

Girardi, L., Bressan, A., Bertelli, G., \& Chiosi, C. 2000, A\&AS, 141, 371

González, J. J. 1993, Ph.D. thesis, Univ. California, Santa Cruz

Gorgas, J., Faber, S. M., Burstein, D., Gonzalez, J. J., Courteau, S., \& Prosser, C. 1993, ApJS, 86, 153

Goto, T., et al. 2003, PASJ, 55, 771

Gratton, R. G., Sneden, C., Carretta, E., \& Bragaglia, A. 2000, A\&A, 354, 169

Grillmair, C. J., et al. 1996, AJ, 112, 1975

Hardy, E., Couture, J., Couture, C., \& Joncas, G. 1994, AJ, 107, 195

Hobbs, L. M., \& Thorburn, J. A. 1991, AJ, 102, 1070

Hurley, J. R., Tout, C. A., Aarseth, S. J., \& Pols, O. R. 2001, MNRAS, 323,630

Jones, L. A. 1999, Ph.D. thesis, Univ. North Carolina

Jones, L. A., \& Worthey, G. 1995, ApJ, 446, L31

Kuntschner, H. 2000, MNRAS, 315, 184

Landsman, W., Bohlin, R. C., Neff, S. G., O'Connell, R. W., Roberts, M. S., Smith, A. M., \& Stecher, T. P. 1998, AJ, 116, 789

Lee, H., Yoon, S., \& Lee, Y. 2000, AJ, 120, 998

Le Fèvre, O., et al. 2001, in Deep Fields, ed. S. Cristiani, A. Renzini, \& R. E. Williams (New York: Springer), 236

Lejeune, Th., Cuisinier, F., \& Buser, R. 1997, A\&AS, 125, 229

Leonardi, A.J., \& Rose, J.A. 2003, AJ, 126, 1811

Maeder, A., \& Meynet, G. 1991, A\&AS, 89, 451

Maraston, C., Greggio, L., Renzini, A., Ortolani, S., Saglia, R. P., Puzia, T. H., \& Kissler-Patig, M. 2003, A\&A, 400, 823

Mathieu, R. D., Latham, D. W., Griffin, R. F., \& Gunn, J. E. 1986, AJ, 92, 1100

Montgomery, K. A., Marschall, L. A., \& Janes, K. A. 1993, AJ, 106, 181 (MMJ)

Nissen, P. E., Twarog, B. A., \& Crawford, D. L. 1987, AJ, 93, 634
O'Connell, R. W. 1980, ApJ, 236, 430

Poggianti, B. M., Bridges, T. J., Komiyama, Y., Yagi, M., Carter, D., Mobasher, B., Okamura, S., \& Kashikawa, N. 2003, ApJ, in press

Puzia, T. H., Saglia, R. P., Kissler-Patig, M., Maraston, C., Greggio, L., Renzini, A., \& Ortolani, S. 2002, A\&A, 395, 45

Rose, J. A. 1984, AJ, 89, 1238

. 1985a, AJ, 90, 787

1985b, AJ, 90, 803

1994, AJ, 107, 206

Sanders, W. L. 1977, A\&AS, 27, 89

Schiavon, R. P. 2004, in preparation

Schiavon, R. P., \& Barbuy, B. 1999, ApJ, 510, 934

Schiavon, R. P., Barbuy, B., \& Bruzual A., G. 2000, ApJ, 532, 453

Schiavon, R. P., Faber, S. M., Castilho, B. V., \& Rose, J. A. 2002a, ApJ, 580,850

Schiavon, R. P., Faber, S. M., Rose, J. A., \& Castilho, B. V. 2002b, ApJ, 580,873

Scott, J. E., Friel, E. D., \& Janes, K. A. 1995, AJ, 109, 1706

Shetrone, M. D., \& Sandquist, E. L. 2000, AJ, 120, 1913

Spinrad, H., \& Taylor, B. J. 1971, ApJS, 22, 445

Tautvaisiene, G., Edvardsson, B., Tuominen, I., \& Ilyin, I. 2000, A\&A, 360,499

Taylor, B. J. 1980, AJ, 85, 242

Trager, S. C., Faber, S. M., Worthey, G., \& González, J. J. 2000, AJ, 120, 165

Tran, K. H., Franx, M., Illingworth, G., Kelson, D., \& van Dokkum, P. 2003, ApJ, 599, 865

Tripicco, M. J., \& Bell, R. A. 1995, AJ, 110, 3035

Vazdekis, A., \& Arimoto, N. 1999, ApJ, 525, 144

Vazdekis, A., Salaris, M., Arimoto, N., \& Rose, J. A. 2001, ApJ, 549, 274

Worthey, G. 2003, AJ, submitted

Worthey, G., Faber, S. M., González, J. J., \& Burstein, D. 1994, ApJS, 94, 687

Worthey, G., \& Ottaviani, D. L. 1997, ApJS, 111, 377

York, D. G., et al. 2000, AJ, 120, 1579 